\title{
Spatiotemporal Variation of Multiple Neurophysiological Signals in the Primary Motor Cortex during Dexterous Reach-to-Grasp Movements
}

\author{
Mohsen Mollazadeh, ${ }^{1}$ Vikram Aggarwal, ${ }^{1}$ Adam G. Davidson, ${ }^{3}$ Andrew J. Law, ${ }^{4}$ Nitish V. Thakor, ${ }^{1}$ \\ and Marc H. Schieber ${ }^{2,3}$ \\ ${ }^{1}$ Department of Biomedical Engineering, Johns Hopkins University, Baltimore, Maryland 21205, and Departments of ${ }^{2}$ Neurology, ${ }^{3}$ Neurobiology and \\ Anatomy, and ${ }^{4}$ Biomedical Engineering, University of Rochester Medical Center, Rochester, New York 14642
}

To examine the spatiotemporal distribution of discriminable information about reach-to-grasp movements in the primary motor cortex upper extremity representation, we implanted four microelectrode arrays in the anterior bank and lip of the central sulcus in each of two monkeys. We used linear discriminant analysis to compare information, quantified as decoding accuracy, contained in various neurophysiological signals. For all signal types, decoding accuracy increased immediately after the movement cue, peaked around movement onset, and declined during the static hold. Spike recordings and local field potential (LFP) time domain amplitude provided more discriminable information than LFP frequency domain power. Discriminable information on movement type was distributed evenly across recording sites by LFP amplitude and $1-4 \mathrm{~Hz}$ power but unevenly by $100-170 \mathrm{~Hz}$ power and spike recordings. These latter two signal types provided higher decoding accuracies closer to the hemispheric surface than deep in the anterior bank and also provided accuracies that varied along the central sulcus. This variation in the distribution of movement-type information may be related to differences in the rostral versus caudal regions of the primary motor cortex and to its underlying somatotopic organization. The even distribution of information by LFP amplitude and 1-4 Hz power compared with the more localized distribution by $100-170 \mathrm{~Hz}$ power and spikes suggest that these different neurophysiological signals reflect different underlying processes that distribute information through the motor cortex during reach-to-grasp movements.

\section{Introduction}

Electrophysiological and anatomical studies have demonstrated at least two types of regional variation within the upper extremity representation of the primary motor cortex (M1, Brodmann's area 4). First, electrical stimulation of M1 in both humans and nonhuman primates has shown some degree of systematic variation in the motor outputs evoked at different cortical sites, summarized as either somatotopic or functional maps (Penfield and Boldrey, 1937; Woolsey et al., 1952; Kwan et al., 1978; Park et al., 2001; Graziano et al., 2002). Second, anatomical, physiological, and functional imaging studies of area 4 have distinguished rostral and caudal regions within the M1 upper extremity representation (see Discussion) (Strick and Preston, 1982a,b; Preuss et al.,

\footnotetext{
Received June 13, 2011; revised Aug. 30, 2011; accepted Sept. 4, 2011.

Author contributions: M.H.S. designed research; A.G.D., A.J.L., and M.H.S. performed research; V.A. and N.V.T. contributed unpublished reagents/analytic tools; M.M. analyzed data; M.M., V.A., and M.H.S. wrote the paper.

This work was funded in part by National Institute of Neurological Disease and Stroke Grant R01 EB010100, the National Science and Engineering Research Council of Canada, DARPA Revolutionizing Prosthetics Program Prime Contract N66001-06-C-8005, and REPAIR Program Prime Contract N66001-10-C-2009. We thank Jay Uppalapati and Andrea Moore for technical assistance, Supratim Ray, Ernst Niebur, and Piotr Franaszczuk for help with MP software, and Marsha Hayles for editorial comments.

Correspondence should be addressed to either of the following: Mohsen Mollazadeh, Department of Biomedical Engineering, Johns Hopkins University, Baltimore, MD 21205; E-mail: mohsenm@jhu.edu; or Marc H. Schieber, Departments of Neurology and Neurobiology and Anatomy, University of Rochester Medical Center, Rochester, NY 14642.E-mail:mhs@cvs.rochester.edu.

DOI:10.1523/JNEUROSCI.2999-11.2011

Copyright $\odot 2011$ the authors $\quad 0270-6474 / 11 / 3115531-13 \$ 15.00 / 0$
}

1997; Binkofski et al., 2002; Sharma et al., 2008; Rathelot and Strick, 2009). The M1 upper extremity representation thus is not homogenous, from either rostral to caudal down the anterior bank of the central sulcus or lateral to medial along the central sulcus.

In contrast, during voluntary movements, little evidence of spatial variation in neural activity has been observed in the macaque M1. Although the upper extremity representation extends $\sim 12 \mathrm{~mm}$ along the central sulcus and lies beneath a pial surface area of $\sim 60 \mathrm{~mm}^{2}$ (Park et al., 2001), kinematics of the entire upper extremity from the shoulder to the hand can be decoded using activity recorded with a $4 \times 4 \mathrm{~mm}\left(\sim 16 \mathrm{~mm}^{2}\right)$ electrode array on the crown of the precentral gyrus (Vargas-Irwin et al., 2010). Similarly, during individuated movement of any digit, active neurons are found distributed $\sim 9 \mathrm{~mm}$ along the central sulcus and $\sim 6 \mathrm{~mm}$ down the anterior bank (Schieber and Hibbard, 1993; Schieber and Rivlis, 2005).

Neural recordings obtained through microelectrode arrays offer the opportunity to examine the spatiotemporal distribution of neural activity during voluntary movements. Most previous studies have used arrays that sampled simultaneously with a limited number of electrodes and/or over a limited M1 territory in any single session (Murthy and Fetz, 1996; Rickert et al., 2005; O'Leary and Hatsopoulos, 2006; Spinks et al., 2008). We therefore implanted four microelectrode arrays that spanned a total of $\sim 12 \mathrm{~mm}$ along the central sulcus and sampled from 1 to $8 \mathrm{~mm}$ 
A

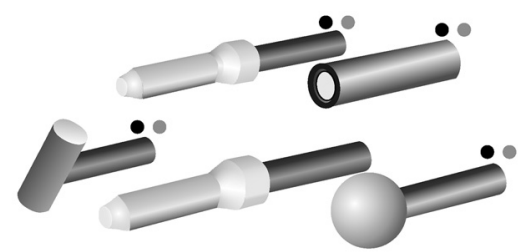

B

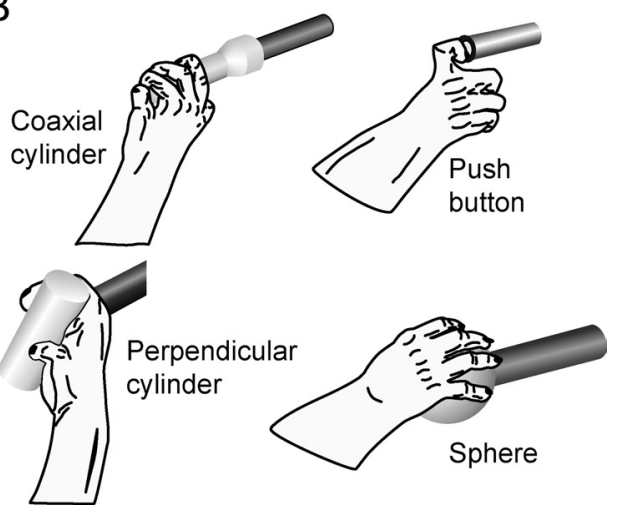

Figure 1. Reach-to-grasp task. $A$, Four peripheral objects and one center object were used in a center-out paradigm. Each trial started when the monkey pulled on the center coaxial cylinder. After a variable initial hold period, a blue LED (dark gray) was illuminated next to one of the peripheral objects, instructing the monkey to reach to and grasp that object. In clockwise order, the objects included a perpendicular cylinder, a coaxial cylinder, a push button, and a sphere. $\boldsymbol{B}$, Drawings from digital video frames illustrate the hand shapes used to grasp the different objects by monkey $X$; similar grasps were used by monkey $Y$. The monkey had to maintain the grasp for a final hold period of $1 \mathrm{~s}$ to receive a food reward.

down the anterior bank from the hemispheric surface in the M1 upper extremity representation of two rhesus monkeys trained to perform dexterous reach-to-grasp movements.

We examined multiple neurophysiological signals recorded from the implanted arrays, including local field potential (LFP) activity in the time and frequency domains, as well as neuron spiking activity. We applied decoding analyses to evaluate the spatiotemporal distribution of movement-type information encoded in each signal type. During reach-to-grasp movements, the entire upper extremity is in motion simultaneously, motion of the digits and wrist preshaping and orienting the hand while motion of the shoulder and elbow transport the hand to the object (Paulignan et al., 1990; Theverapperuma et al., 2006). Hence, we expected that discriminable information about movement type would be distributed evenly throughout the M1 upper extremity representation.

\section{Materials and Methods}

All procedures involving nonhuman primates were approved by the University Committee on Animal Resources at the University of Rochester.

Behavioral tasks. Two male Rhesus monkeys (Macaca mulatta; monkey Y, $9 \mathrm{~kg}$; monkey X, $8 \mathrm{~kg}$ ) were trained to perform a center-out, reach-to-grasp task with the right hand, the left upper extremity being restrained within the primate chair. The monkey viewed a central home object and four peripheral objects arranged at $45^{\circ}$ intervals at a radius of $13 \mathrm{~cm}$ from the home object (Fig. 1A). Each object was mounted on its own horizontal rod projecting toward the monkey.

A trial began when the monkey grasped the central cylinder (mounted coaxially on its mounting rod, which pointed directly at the monkey's right shoulder) and pulled the cylinder toward itself $\sim 1 \mathrm{~cm}$ against a small spring load. After a variable initial hold period during which the monkey maintained its pull on the central cylinder, a blue LED was illuminated next to the rod supporting one of the four peripheral objects. (The range of the initial hold period duration differed among sessions:
Y0225, 1034-1531 ms; Y0228, 1034-1533 ms; X0917, 329-628 ms; X0918, 579-878 ms.) Illumination of a blue LED (cue) instructed the monkey to promptly release the central object and then reach to and grasp the indicated peripheral object. Release of the central object was considered the onset of movement (OM). After grasping the peripheral object, the monkey was required to rotate the sphere $45^{\circ}$, to pull the perpendicular cylinder, to depress the push button ( $12 \mathrm{~mm}$ diameter), or to pull the peripheral coaxial cylinder, each against a small spring load (Fig. 1B). Appropriate manipulation of each object closed a microswitch, which was indicated to the monkey by illumination of a green LED, also mounted next to the rod supporting the peripheral object. The switch closure marked the beginning of a static hold ( $\mathrm{SH}$ ), during which the monkey was required to maintain the object in its final position for 1000 $\mathrm{ms}$. Thereafter, the blue LED was turned off. Such trials were considered successful, and the monkey received a food pellet reward (Bioserv Biotechnologies). After successful completion of a trial, the monkey was free to release the peripheral object and initiate another trial by again pulling on the central coaxial cylinder.

The instructed objects were presented in a pseudorandomized block design. Trials were aborted immediately as errors if the monkey released the central coaxial cylinder before illumination of a blue LED, manipulated a non-instructed object, or released the instructed object before completion of the final hold period. Error trials were repeated until successfully completed. Averaged across all successful trials, reaction time (from the cue to the $\mathrm{OM}$ ) was $343 \pm 81 \mathrm{~ms}$ (mean $\pm \mathrm{SD}$ ) for monkey $\mathrm{Y}$ and $250 \pm 72 \mathrm{~ms}$ for monkey X. Movement time (from the OM to the beginning of the $\mathrm{SH}$ ) averaged $471 \pm 281 \mathrm{~ms}$ for monkey Y and $289 \pm$ $122 \mathrm{~ms}$ for monkey X. Monkey Y thus tended to react and move more slowly than monkey $\mathrm{X}$, although the experimental setup was identical for both monkeys.

Microelectrode array implantation. Using sterile technique and isoflurane anesthesia, each monkey was implanted with multiple floating microelectrode arrays (FMAs; MicroProbes for Life Sciences) in cortical motor areas of the left hemisphere. Because the length of each electrode on an FMA can be specified from 1 to $10 \mathrm{~mm}$ at the time of manufacture (Musallam et al., 2007), each of the FMAs we used incorporated electrodes of various lengths so as to sample neural activity at different depths down the anterior bank of the central sulcus. Each FMA consisted of 16 parylene-C insulated platinum/iridium recording microelectrodes of different lengths, varying from 1.5 to $8.0 \mathrm{~mm}$ in monkey $\mathrm{Y}$ and from 1.0 to $6.0 \mathrm{~mm}$ in monkey $\mathrm{X}$, arranged in a $4 \times 4$ triangular matrix on a $1.95 \times$ $2.45 \mathrm{~mm}$ ceramic chip. Two additional low impedance microelectrodes on each array served as reference and ground electrodes. After craniotomy and durotomy, each FMA was advanced slowly into the cortex at a location selected based on direct visualization of the hemispheric surface (Fig. 2A).

After all arrays had been implanted, the dura mater was closed loosely and covered with Duragen (Integra), after which the craniotomy was closed with methylmethacrylate. Array connectors were imbedded in additional methylmethacrylate, and a polycarbonate chamber was mounted over the array connectors. The entire implant was fixed to the skull with circumferentially placed titanium bone screws also embedded in methylmethacrylate, along with a head-holding post. Postoperatively, each monkey received a $3 \mathrm{~d}$ course of banamine $\left(1.1 \mathrm{mg} \cdot \mathrm{kg}^{-1} \cdot \mathrm{d}^{-1}\right.$, i.m.) for pain and a $2-6$ week course of ceftriaxone $\left(50 \mathrm{mg} \cdot \mathrm{kg}^{-1} \cdot \mathrm{d}^{-1}\right)$ for infection prophylaxis and was maintained for several weeks on phenytoin $\left(10 \mathrm{mg} \cdot \mathrm{kg}^{-1} \cdot \mathrm{d}^{-1}\right)$ for seizure prophylaxis. After a recovery period of at least 1 week, each monkey returned to performing the behavioral task described above in daily sessions, now with the head fixed.

Acquisition of neurophysiological signals. Neuron spikes and LFP activity were recorded using a Plexon data acquisition system. Signals from the microelectrodes (impedance, $\sim 0.5 \mathrm{M} \Omega$ ) were amplified $20 \times$ by a head stage and then hardware filtered separately for LFPs $[0.7 \mathrm{~Hz}$ (twopole) to $175 \mathrm{~Hz}$ (four-pole)] and spikes [100 $\mathrm{Hz}$ (two-pole) to $8 \mathrm{kHz}$ (four-pole)]. LFPs from every other electrode (total of 32 channels) were then hardware amplified $50 \times$ and digitized at $1 \mathrm{kHz}$ through a National Instruments PXI-6071 analog-to-digital converter at a total amplification of $1000 \times$. LFPs from five channels in monkey $Y$ and three channels in monkey $\mathrm{X}$ were found to have either excessively high noise or no signal 
A

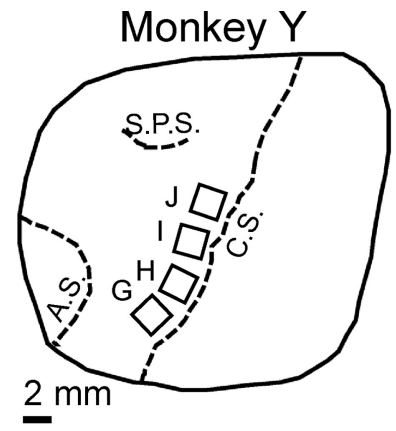

B

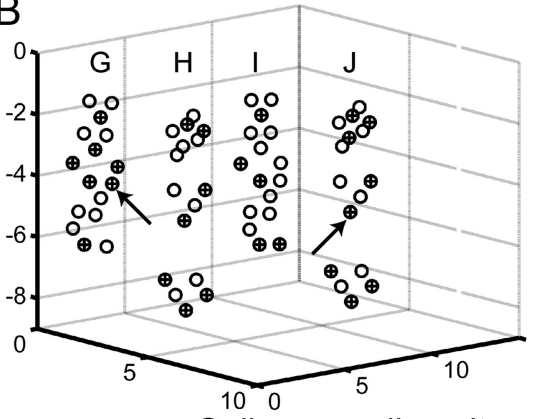

C

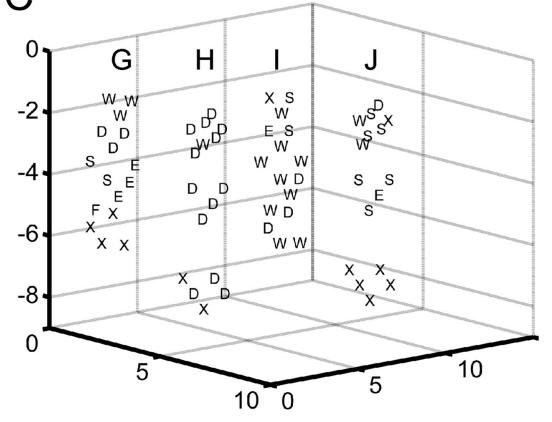

$\mathrm{S}=$ shoulder $\mathrm{E}=$ =elbow $\quad \mathrm{W}=$ wrist
Monkey X
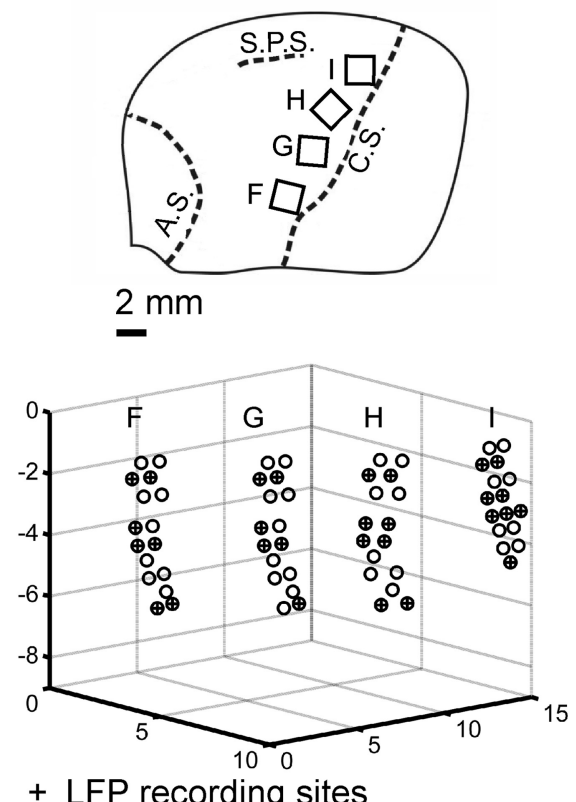

+ LFP recording sites

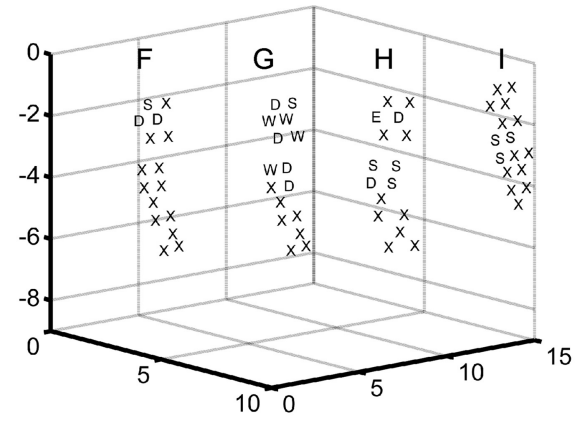

$D=$ digits $\quad F=$ face $\quad X=$ no response

Figure 2. Recording locations. $\boldsymbol{A}$, Drawings, traced from intraoperative photographs, show the location of floating microelectrode arrays (rectangles) implanted in monkey Y (left column) and in monkey X (right column) relative to three sulci (dashed lines): C.S., Central sulcus; A.S., arcuate sulcus; S.P.S., superior precentral sulcus. Letters G-J designate individual arrays in monkey $Y$, and letters F-I designate individual arrays in monkey X. $\boldsymbol{B}$, Three-dimensional plots for each monkey show the relative location of the tip of each recording electrode (circles), estimated from the location and orientation of the parent array in the intraoperative photograph, and the known length of each electrode. The plots are oriented as if the reader is viewing the anterior bank of the central sulcus from the posterior bank, with anterolateral to the left, posteromedial to the right, and the hemispheric surface at the top. Scales are in millimeters. Spikes were recorded from all electrodes, whereas LFPs were recorded from every other electrode. Electrodes used to record LFPs are indicated with a + symbol filling the circle. The LFPs shown in Figures 3 and 4 were obtained from the two electrodes indicated here with arrows. $C$, Letters indicating the body part that moved in response to threshold ICMS have been plotted at the location of each electrode tip. All four arrays in each monkey were within the M1 upper extremity representation. Although no response was evoked from many recording sites with currents up to $40 \mu \mathrm{A}$, the responses obtained here were consistent with the nested-horseshoe somatotopic organization described by others (see Materials and Methods).

and therefore were discarded from analysis. The electrodes that provided the remaining LFPs for analysis are filled with a + sign inside the circle representing each electrode tip location in Figure $2 \mathrm{~B}$. Neuron spiking activity from each of the 64 recording electrodes was amplified from 1000 to 32,000 times, and waveforms crossing a threshold selected online by an investigator were sampled at $40 \mathrm{kHz}$ and saved for offline sorting, which was performed with Offline Sorter by Plexon. After offline sorting, spike clusters with a waveform signal-to-noise ratio (SNR) $>3.0$ and with no interspike intervals (ISIs) of $1 \mathrm{~ms}$ or less were considered well-isolated single-unit (SU) recordings, whereas spike clusters with SNR $\leq 3$ or ISIs $\leq 1$ ms were considered multiunit (MU) recordings.

Location of recording electrodes. The present report focuses on recordings obtained from four FMAs implanted just anterior to the central sulcus in each monkey, such that their microelectrodes entered the cortex up to 2.5 $\mathrm{mm}$ anterior to the sulcus and also penetrated down the anterior bank of buried cortex in the M1 upper extremity representation. Figure 2 shows for each monkey the location of these arrays redrawn from intraoperative photographs $(A)$, as well the relative electrode tip locations estimated from the intraoperative photographs and electrode lengths for each array $(B)$. Note that, in estimating these electrode tip locations, the bases of all four arrays were assumed to lie in the same plane.

Several months after the present recording sessions had been completed, intracortical microstimulation (ICMS) was performed through each recording electrode. Before ICMS sessions, the monkey's right side was shaved to facilitate identification of small muscle twitches. At the beginning of each ICMS session, the impedance of each recording electrode was measured (range, $0.4-2.7 \mathrm{M} \Omega$ at 1 $\mathrm{kHz}$ using an 18-channel impedance tester; MicroProbes for Life Sciences) to confirm that the electrode and current path remained intact. The monkey was lightly tranquilized with 5 $\mathrm{mg} / \mathrm{kg}$ ketamine, and $0.04 \mathrm{mg} / \mathrm{kg}$ atropine was given to reduce secretions; both medications were repeated every $30-60 \mathrm{~min}$ as needed to maintain a tranquil state in which weak muscle twitches could be identified. ICMS was performed as the monkey sat in a primate chair with the right upper arm resting at its side and the elbow flexed to $90^{\circ}$ with the forearm resting on a support. Conventional trains of 12 biphasic ( $0.2 \mathrm{~ms}$ per phase) constant current pulses at $333 \mathrm{~Hz}$ were delivered at $3 \mathrm{~s}$ intervals via an optically isolated stimulator (BAK BSI-1). Stimulating current was monitored continuously via a high impedance amplifier (WPI DAM80) as the voltage drop across a $100 \Omega$ series resistor. Current was gradually increased until a muscle twitch or an overt movement was observed by one investigator and confirmed by another or until a maximum of 40 $\mu \mathrm{A}$ was reached. Threshold was measured as the current at which a response was evoked by $50 \%$ of stimulus trains. Each muscle twitch or movement evoked at threshold was assigned to one of the following categories: digits, wrist, elbow, shoulder, face, axial, or leg.

Figure $2 C$ shows the responses to ICMS evoked in each monkey. No response was obtained from several electrodes that presumably were not close enough to cortical layer $\mathrm{V}$ for a response to be evoked at $40 \mu \mathrm{A}$. ICMS confirmed that all four arrays in each monkey were situated in the M1 upper extremity representation. Furthermore, the responses obtained in both monkeys were consistent with the nested-horseshoe somatotopic organization of the upper extremity representation as defined with ICMS in previous studies of macaque M1 (Kwan et al., 1978; Park et al., 2001). A core of distal, digit representation extending down the anterior bank of the central sulcus in each monkey was flanked medially by progressively more proximal representation and was flanked laterally by a narrower region of proximal representation.

Both monkeys remain alive at this time. Histological confirmation of recording sites and the cortical lamina in which each electrode tip was located therefore were unavailable.

Power spectral estimation. Time-frequency analysis of LFPs was performed with a matching pursuit (MP) algorithm (Mallat and Zhang, 
1993), using software available from http://erl.neuro.jhmi.edu/mpsoft and as described in detail by Ray et al. (2008). MP is an iterative process that adaptively decomposes the signal as a linear combination of functions $g_{\gamma \mathrm{n}}$ belonging to a large overcomplete dictionary. Here, we used "Gabor atoms" or "Gabor functions," which are sine-modulated Gaussian functions that provide the best time-frequency resolution, in addition to Dirac $\delta$ functions and Fourier atoms. The MP algorithm reiteratively projects the signal onto an atom that best describes the signal, $g_{\gamma \mathrm{n}}$ (i.e., which has the highest inner product with the signal) and replaces the signal with the difference between the signal and the projection (residue). Unlike fast Fourier transform, multitapering methods, or empirical mode decomposition, MP does not use only oscillatory functions with a fixed temporal support and hence can represent the sharp transients in LFP signals with functions that have short temporal support (Ray et al., 2008). Because line noise is represented by atoms localized around $60 \mathrm{~Hz}$ and its harmonics and is spread over time, such atoms were removed from analysis to exclude $60 \mathrm{~Hz}$ artifact. Time-frequency plots were obtained by calculating the Wigner distribution of each atom and taking the weighted sum across all atoms (Mallat and Zhang, 1993).

Using the MP algorithm, we examined LFP activity from $-1.047 \mathrm{~s}$ before to $1 \mathrm{~s}$ after three behavioral events: illumination of a blue LED (cue); OM, when the monkey released the center object; and the beginning of the SH. All post-MP decomposition computations were performed using custom code written in MATLAB (MathWorks). The MP decomposition yielded a 2048 (time) $\times 1024$ (frequency) array of timefrequency values with a temporal resolution of $1 \mathrm{~ms}$ and frequency resolution of $\sim 0.5(1000 / 2048) \mathrm{Hz}$. This data array was further downsampled by a factor of 4 , yielding a temporal resolution of $4 \mathrm{~ms}$ and frequency resolution of $\sim 2 \mathrm{~Hz}$.

With LFP data aligned on cue, OM, or $\mathrm{SH}$, power in a given time window was computed by averaging the energy within that time period ( $T$ ) at a given frequency.

$$
P(\omega)=\frac{1}{T} \sum_{t_{0}}^{t_{0}+T} E(t, \omega),
$$

where $E(t, \omega)$ is the energy at time $t$ and frequency $\omega$ obtained from the MP algorithm. Time-frequency plots (see Fig. 4) were calculated by subtracting the power at each time point from the baseline power:

$$
D(t, \omega)=10 \times\left(\log _{10} E(t, \omega)-\log _{10} B(\omega)\right),
$$

where $B(\omega)$ is the baseline energy computed with Equation 1 using $t_{0}=$ $-256 \mathrm{~ms}, T=256 \mathrm{~ms}$, and data aligned on the cue, i.e., power during the $256 \mathrm{~ms}$ period immediately preceding the cue. LFP power for each channel at each time window and frequency point $[P(\omega)]$ was normalized to zero mean and unit SD across all trials. For additional analyses, LFP activity was divided into seven frequency bands $(1-4,5-13,16-24,25-$ $40,41-59,62-98$, and $100-175 \mathrm{~Hz}$ ), and the power was averaged across each band after it was normalized relative to baseline $=1$. These bands were chosen based on uniformity of power modulation within these ranges over different recording sites and monkeys in the present study.

Movement decoding using LFPs. Linear discriminant analysis (LDA) was used to decode movement type, using features from LFPs or spike recordings. Data from all trials in a given recording session were pooled and randomly assigned to non-overlapping training and testing sets. In each case, LDA was repeated 20 times, randomly selecting LFP channels or spike recordings for the training and testing sets. The conditional probability of the features belonging to a certain class can be defined as follows:

$$
\begin{aligned}
& f_{i}(x)=x^{T} \sum^{-1} \mu_{i}-\frac{1}{2} \mu_{i}^{T} \sum^{-1} \mu_{i}+\ln \left(p_{i}\right) \\
& C(x)=\arg \max _{i} f_{i}(x)
\end{aligned}
$$

where $x$ is the input feature set, $\sum$ is the pooled within group covariance matrix, $\mu_{i}$ is the mean and $p_{i}$ is the prior estimate for the $i$ th group, and $f_{i}$ is the conditional probability of $x$ being in class $i$. During testing, the decoded output class $(C)$ was selected based on the highest conditional
Table 1. Number of spike recordings, LFP channels, and successful trials in each session

\begin{tabular}{llll}
\hline Recording session & Spike recordings (SU + MU) & LFP channels & Number of trials \\
\hline Y0225 & $57(28+29)$ & 27 & 560 \\
Y0228 & $73(43+30)$ & 27 & 625 \\
X0917 & $51(14+37)$ & 29 & 884 \\
X0918 & $58(18+40)$ & 29 & 629 \\
\hline
\end{tabular}

For spike recordings, the total number is given, as well as the number of SU and MU recordings (in parentheses).

probability. For LFP activity, the power in each of the seven frequency bands ( $T=256 \mathrm{~ms}$, Eq. 1$)$ as well as the amplitude in the same time window was used as the set of features for LDA. For spiking activity, the mean firing rate during the same $256 \mathrm{~ms}$ windows (centered on each time point) was used for decoding. As detailed in Results, multiple feature sets with varying numbers of LFP channels or spike recordings were created to observe the effects of various factors on decoding accuracy. In each case, the LDA was trained using features in successive overlapping time windows of $250 \mathrm{~ms}$ duration, sliding every $50 \mathrm{~ms}$. To examine the timedependent variation in neural signals related to different behavioral task events, we performed similar analysis with the data aligned separately for each behavioral marker: cue, OM, and $\mathrm{SH}$.

\section{Results}

We recorded spike and LFP data from microelectrode arrays permanently implanted in each of two monkeys performing reachto-grasp movements. Two sessions were analyzed from each monkey to ensure that the findings reported here were not idiosyncratic to a particular session in either monkey. Table 1 summarizes the number of SU and MU spike recordings, LFP channels, and successful trials analyzed in each of the two sessions from each monkey. Whereas for monkey Y all four movement types were incorporated in the analyses described below, a noise transient occurred whenever monkey $\mathrm{X}$ grasped the peripheral coaxial cylinder, and for monkey $\mathrm{X}$, we therefore excluded this movement type from analysis.

\section{LFP activity in the time domain}

To examine LFP modulation in the time domain, we formed motor evoked potentials (mEPs) for each channel in each recording session by averaging LFP amplitude across the multiple successful trials of a given movement type with the data aligned on a particular behavioral event. Figure 3 illustrates such mEPs for one channel from array G (top row) and one from array J (bottom row), averaged across all correctly performed trials of each of the four movement types in session Y0225. Separate mEPs for each movement type (sphere, blue; perpendicular cylinder, green; push button, red; and coaxial cylinder, cyan) were formed for data aligned at the times of the cue (Fig. 3A), the OM (Fig. 3B), and the beginning of the $\mathrm{SH}$ (Fig. $3 C$ ). In each frame, a solid vertical line marks the time of alignment, and dashed vertical lines mark the average times of the other two behavioral events.

Although in each frame the $\mathrm{mEP}$ waveforms for different movement types generally were similar, separation of the traces indicated that, within a single channel, mEPs varied depending on the movement performed. The same was true of population average mEPs (data not shown). Variation in mEPs related to movement type tended to be least immediately after the cue, slightly greater by the $\mathrm{OM}$, and greatest around the time of $\mathrm{SH}$. All mEPs showed significant movement type (four or three levels for monkey $\mathrm{Y}$ or $\mathrm{X}$, respectively) $\times$ time (three levels; cue, $\mathrm{OM}$, $\mathrm{SH}$ ) interactions (two-way ANOVA, $p<0.01$ ), indicating that, at some time point(s) during the trials, the amplitude of each LFP recording varied depending on the movement type. 
A

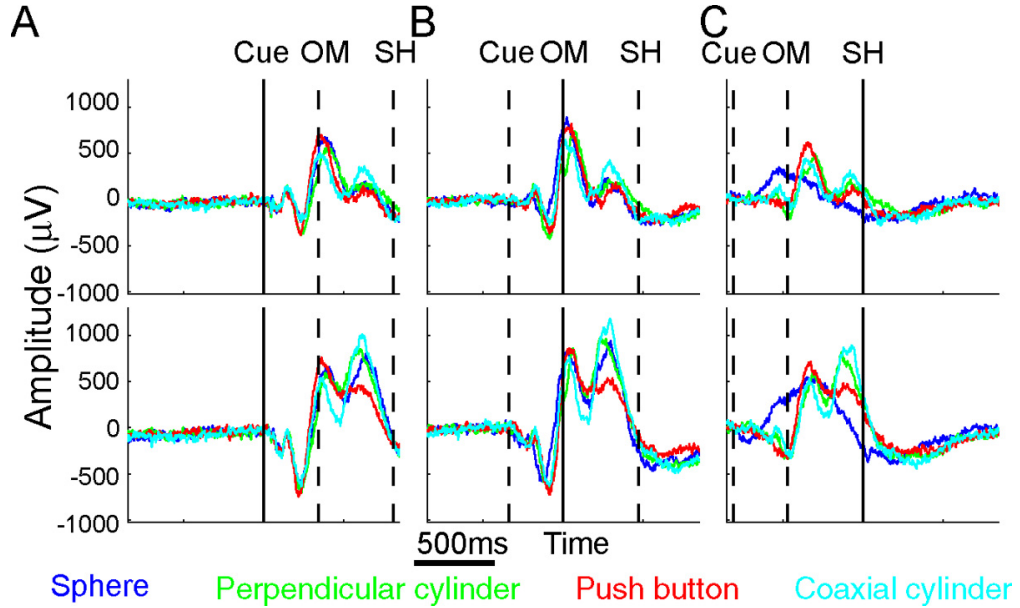

Figure 3. Variation in mEPs. LFP amplitude in the time domain was averaged across all successful trials of each movement type to generate $m E P$ s for two channels from the same recording session in monkey $Y$ : one channel in array $G$ (top) and another in array $\mathrm{J}$ (bottom) indicated with arrows in Figure 2. Separate mEPs were formed by averaging data aligned at the cue $(\boldsymbol{A}), 0 \mathrm{M}(\boldsymbol{B})$, or SH (C). The $\mathrm{mEPs}$ in the two channels differed in many respects but had a number of general similarities. An early negative trough and positive peak were evident shortly after the cue. $0 \mathrm{M}$ was preceded by a second negative peak. By the time of $\mathrm{SH}$, the average traces in both monkeys were significantly different depending on the object. Similar results could be observed in population averages for all recording sessions (data not shown). The modulation of LFP amplitude in all recorded channels showed significant movement type $\times$ time interactions (two-way ANOVA, $p<0.01$ ).

For a given movement type, mEP waveforms were generally similar in the two electrodes from different arrays shown in Figure 3, but differences were observed as well. During movements to the push button (red), for example, positive peaks occurred just after the $\mathrm{OM}$ and again before the $\mathrm{SH}$, but the latter peak was relatively larger in the electrode from array J (bottom) than in that from array G (top). Such differences between electrodes suggested some degree of spatial variation in mEPs within the M1 upper extremity representation.

\section{LFP activity in the frequency domain}

To examine LFP modulation in the frequency domain, we formed separate time-frequency plots for each channel in each recording session. Time-frequency plots for each movement type were formed separately with the data aligned on each behavioral event. Figure 4 illustrates such plots of LFP activity for the same two single channels in the arrays $\mathrm{G}$ and $\mathrm{J}$ of monkey $\mathrm{Y}$ from which the mEPs are shown in Figure 3. Here, separate time-frequency plots are shown for each movement type, all aligned at the OM (solid vertical line), with dashed vertical lines marking the average times of the cue and SH. These time-resolved power spectra showed a strong increase in LFP power in the $1-13 \mathrm{~Hz}$ range that began promptly after the cue, continued through the OM, and then declined between OM and SH. A broader increase in LFP power in the $60-170 \mathrm{~Hz}$ range began after the cue but before the $\mathrm{OM}$ and continued with variation in intensity to the beginning of the $\mathrm{SH}$, during which time the monkey reached to and grasped the target object and then declined afterward. In contrast, power in the $16-40 \mathrm{~Hz}$ range decreased before the $\mathrm{OM}$ and returned to baseline only several hundred milliseconds after the beginning of the $\mathrm{SH}$. The decrease in $16-40 \mathrm{~Hz}$ power was stronger in monkey $\mathrm{X}$ than in monkey $\mathrm{Y}$ (data not shown). Similar results were observed in the population averages.

In each channel, although similar patterns of LFP power modulation occurred during movements to the different objects, variation related to the different movement types also was evident. At the OM, power in the $1-13 \mathrm{~Hz}$ range was particularly strong when movements were made to the perpendicular cylinder, for example, whereas $\sim 100$ $\mathrm{ms}$ before the beginning of the $\mathrm{SH}$ power in the $60-170 \mathrm{~Hz}$ range showed a short burst when movements were made to the sphere. Furthermore, the time-resolved power spectra differed between channels. The burst of power in the $60-170 \mathrm{~Hz}$ range around the $\mathrm{OM}$ was stronger in the electrode from array J (bottom) than in that from array G (top), for example, and was relatively more intense for movements to the sphere than for other movement types.

To examine frequency-dependent variation in greater detail, for each movement type, we subdivided the frequencies from $1-170 \mathrm{~Hz}$ into seven bands and plotted normalized power in each band (relative to a baseline of 1) as a function of time. Figure 5 plots such normalized LFP power as a function of time for data averaged over the eight channels from one array in monkey $\mathrm{Y}($ array $\mathrm{H})$ and one in monkey $\mathrm{X}$ (array $\mathrm{H}$ ) in a single session from each monkey. Similar plots were generated for each array in each session to compare more quantitatively how the movement-type related variation in LFP power was modulated depending on the frequency band.

Movement-related changes in normalized power were largest in the $1-4 \mathrm{~Hz}$ band in both monkeys, increasing up to fourfold by the onset of some movements. Early increases also occurred in power in the $5-13 \mathrm{~Hz}$ band, sometimes larger than twofold, although these increases tended to be stronger in monkey $\mathrm{X}$ than in monkey Y. (Note that, because the calculation of power at each time point included data from $125 \mathrm{~ms}$ before to $125 \mathrm{~ms}$ after that time point, even the time point nominally $50 \mathrm{~ms}$ before the cue incorporated data from up to $75 \mathrm{~ms}$ after the cue and hence could be significantly different from baseline.) Power in the 16-24 and $25-40 \mathrm{~Hz}$ bands decreased before the OM. These decreases were deeper, faster, and returned to baseline more quickly in monkey $\mathrm{X}$ than in monkey Y. Also, compared with other bands, normalized power in the 16-24 and $25-40 \mathrm{~Hz}$ bands showed relatively little variation depending on movement type. Power in the 41-59 $\mathrm{Hz}$ bands was relatively flat in both monkeys. In the 62-98 and $100-170 \mathrm{~Hz}$ bands, however, normalized power again showed up to twofold increases by the OM. Movement-dependent variation in these high-frequency bands persisted longer into the final $\mathrm{SH}$ period than in the low-frequency $1-4$ and $5-13 \mathrm{~Hz}$ bands. Overall, larger modulation of normalized LFP power, with more variation related to movement type, appeared in the lower-frequency (1-4 and 5-13 Hz) and the higher-frequency (62-98 and $100-$ $170 \mathrm{~Hz})$ bands than in mid-frequency $(16-24,25-40$, and $41-59$ $\mathrm{Hz}$ ) bands. Similar results were also observed for the other arrays in both recording sessions from each monkey.

\section{Neuron spiking activity}

As enumerated in Table 1, more spike recordings were available than LFP channels in each recording session. Because previous studies have indicated that MU recordings can provide decoding of direction equivalent to that of SUs (Liu and Newsome, 2006; Chestek et al., 2009), in the present analysis, we treated SU and MU spike recordings equivalently. Figure 6 illustrates spike re- 
A

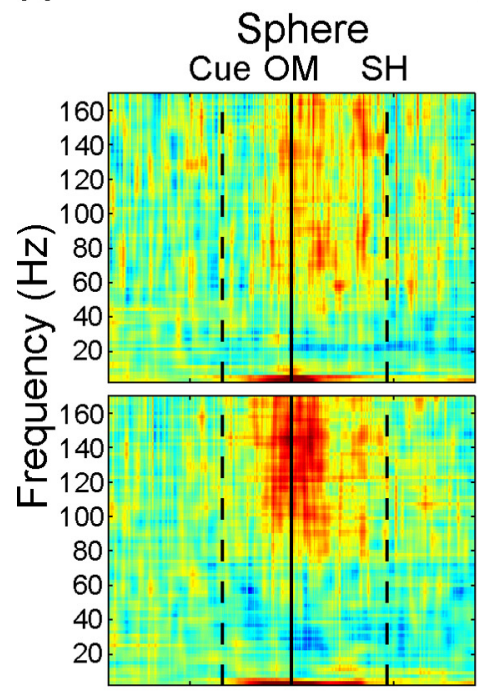

B Perpendicular cylinder
Cue OM SH

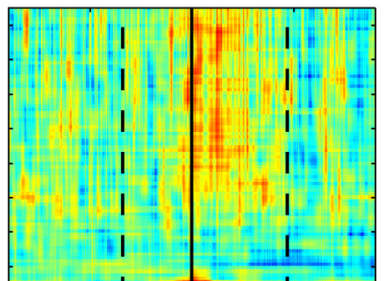

C (a)

Push button Cue OM SH
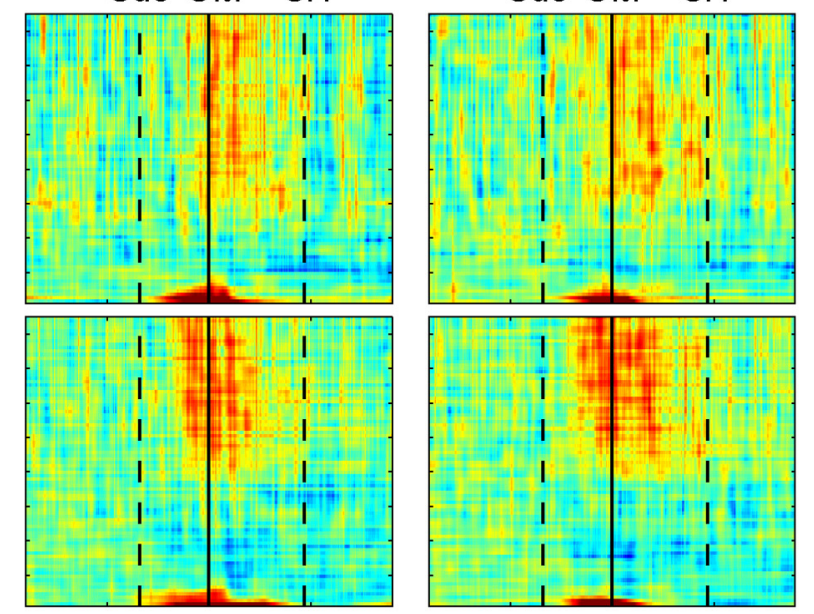

$500 \mathrm{~ms}$ Time

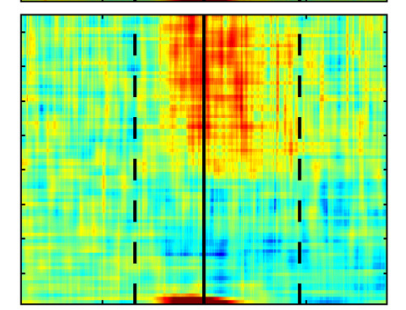

D Coaxial cylinder Cue OM SH
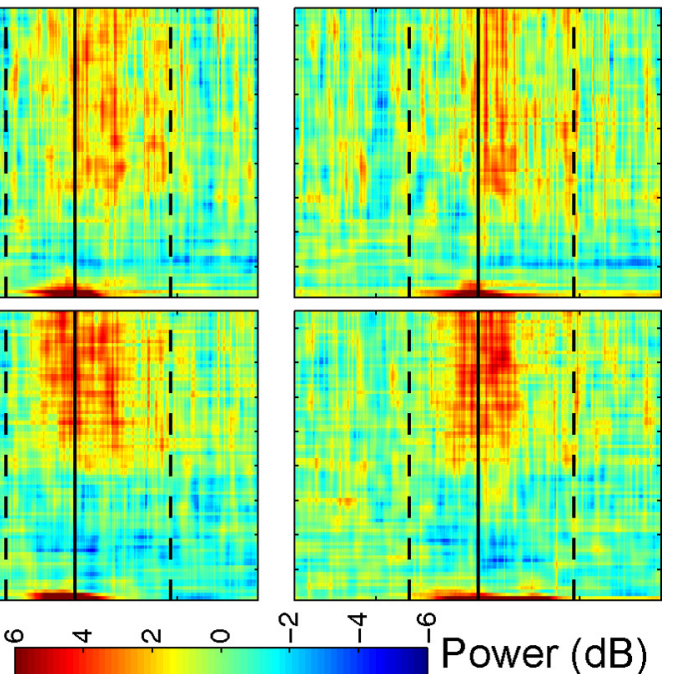

Figure 4. Variation in frequency domain power: time-frequency plots. Single-channel LFP power relative to baseline is plotted (color scale) as a function of time (abscissa) and frequency (ordinate) for the same two channels (top and bottom rows) in the same session as those illustrated in Figure 3. Data aligned at the $0 \mathrm{M}$ (solid vertical line) are shown separately for each of the four objects $(\boldsymbol{A}-\boldsymbol{D})$. Times of the cue and SH averaged across all movement types are indicated by dashed vertical lines. As in the time domain, the two channels differed in many respects but had a number of general similarities. A large increase in low-frequency power was evident shortly after the cue and varied depending on the object. Increase in power at frequencies above $60 \mathrm{~Hz}$ began before the OM and was sustained until the SH. The decrease in power in the $15-40 \mathrm{~Hz}$ range was generally small in monkey $\mathrm{Y}$, illustrated here, but was more prominent in monkey $\mathrm{X}$ (data not shown).

cordings from two SUs recorded in session Y0225, one from array $\mathrm{H}$ (2.0 mm deep to the hemispheric surface) and one from array I (1.5 mm deep). Histograms are shown for each movement type aligned separately at the cue, $\mathrm{OM}$, and $\mathrm{SH}$. To permit more direct comparison with frequency domain LFP activity (Fig. 4), these histograms were formed using the firing rate averaged in a $250 \mathrm{~ms}$ window centered at each $1 \mathrm{~ms}$ time step. Population averages showed that, like the two units illustrated in Figure 6, spiking activity generally increased promptly after the cue, reached nearmaximal levels by the OM, and was declining by the time of the $\mathrm{SH}$, with variation depending on movement type (data not shown). The two SUs illustrated in Figure 6 showed many similarities, discharging a short burst during movements to the coaxial cylinder (cyan), for example, but showing more sustained activity during movements to the push button (red). Clear differences between the two units were present as well. During movements to the push button (red), for example, the firing rate of the SU from array $\mathrm{H}$ (top) showed an initial peak before the OM, which was not present in the SU from array I (bottom).

\section{Comparing different neurophysiological signals}

The neurophysiological signals examined here-LFP activity in the time domain, LFP activity in the frequency domain, and neuron spiking activity_-are not directly comparable. Nevertheless, each signal type varied depending on the movement performed and therefore could contain discriminable information on movement type. We therefore applied LDA to compare the extent to which different movement types could be discriminated using the different neurophysiological signals, quantifying the discriminable information available in each as decoding accuracy. Figure 7 illustrates decoding accuracy in the $250 \mathrm{~ms}$ centered on the beginning of the $\mathrm{SH}$ as a function of the number of channels for each type of signal. For all signal types, decoding accuracy typically increased toward an asymptote as more LFP channels or spike recordings were included.
For LFP activity in the time domain (Fig. $7 A$ ), very similar decoding accuracies were obtained in the two sessions from each monkey, but decoding accuracies were systematically lower for monkey Y. With five channels, for example, decoding accuracy at the beginning of the $\mathrm{SH}$ in monkey $\mathrm{X}$ was $\sim 74 \%$ but in monkey $\mathrm{Y}$ was only $\sim 52 \%$, rising with 20 channels to $\sim 95$ and $\sim 68 \%$ in monkeys $\mathrm{X}$ and $\mathrm{Y}$, respectively. One might attribute this discrepancy to the larger number of movements being decoded in monkey Y (four, chance level of 25\%) than in monkey X (three, chance level of 33\%). We therefore recomputed decoding accuracy for monkey Y's two sessions using only the same three movement types decoded in monkey $\mathrm{X}$ (dotted curves in Fig. 7A). Although this increased the decoding accuracies for monkey $\mathrm{Y}$, superior decoding accuracy still was obtained in monkey $\mathrm{X}$ using LFP amplitude in the time domain.

In the frequency domain, LFP power in different bands provided different levels of decoding accuracy. As illustrated for session X0918 in Figure 7B, decoding accuracy typically was highest in two bands: $1-4$ and $100-170 \mathrm{~Hz}$. Although modulation of LFP power also was substantial in the 5-13 and 62-98 Hz bands (Fig. 5 ), across sessions these two bands provided lower decoding accuracies than the 1-4 and $100-170 \mathrm{~Hz}$ bands (data not shown). The 16-24, 25-40, and 41-59 Hz bands consistently provided the lowest decoding accuracies, often little better than chance even when using all channels. In additional analyses (below), we therefore focused on LFP power in the 1-4 and 100-170 Hz bands, treating each as a separate neurophysiological signal.

Even using these two frequency bands, decoding accuracies using LFP power in the frequency domain were lower than those obtained using LFP amplitude in the time domain. For example, decoding accuracies of $\sim 52 \%$ were obtained with five channels using either $1-4$ or $100-170 \mathrm{~Hz}$ power, whereas accuracies of $\sim 74 \%$ were obtained with five channels in the time domain. The same was true with larger numbers of channels. With 25 channels, for example, accuracies of $\sim 72$ and $\sim 96 \%$ were obtained in 
A

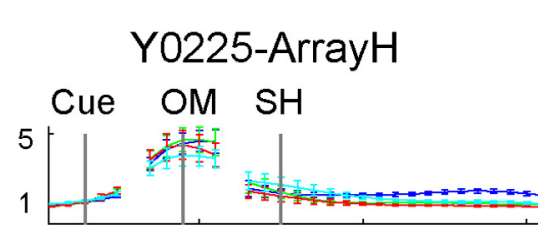

B
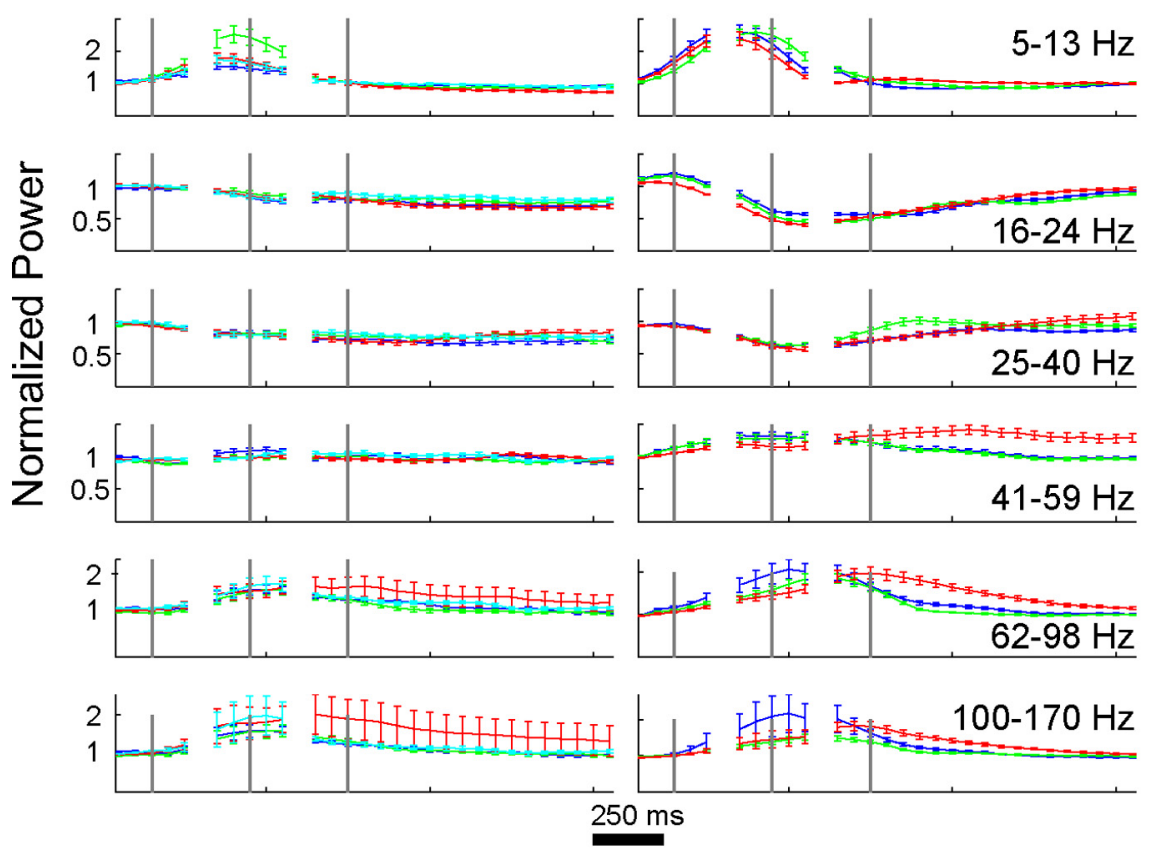

Sphere

Perpendicular cylinder

Push button

Figure 5. Modulation of LFP power in seven frequency bands. LFP power in each of seven frequency bands averaged across the eight LFP channels from a single array in each monkey $(\boldsymbol{A}$, monkey $Y ; \boldsymbol{B}$, monkey $\mathrm{X})$ and normalized relative to baseline $(=1)$ is shown as a function of time for each of the movements performed by the monkey. For each movement, average power was computed at $50 \mathrm{~ms}$ intervals for data aligned separately on the cue, $0 \mathrm{M}$, and SH. Values plotted are the mean $\pm \mathrm{SE}$ across the eight channels in the array. Movement-related changes in normalized power were largest in the 1-4,5-13,62-98, and $100-170 \mathrm{~Hz}$ bands. The low-frequency increases tended to peak around $0 \mathrm{M}$, whereas high-frequency increases tended to peak between $0 \mathrm{M}$ and SH. Power in the $16-24$ and $25-40 \mathrm{~Hz}$ bands decreased more prominently in monkey $\mathrm{X}$ than in monkey $\mathrm{Y}$.

the frequency and time domains, respectively. Decoding accuracy thus was somewhat lower in the frequency domain than in the time domain.

Figure 7C illustrates decoding accuracy as a function of the number of spike recordings used, including both SU and MU recordings. Although $>30$ spike recordings were available in each session, decoding accuracies using features from 30 spike recordings were already close to $100 \%$, and the illustrated curves therefore have been truncated at this point. Despite the difference in chance levels between the monkeys (25\% for monkey Y; $33 \%$ for monkey X), these curves were remarkably similar for both sessions from the two monkeys. In both monkeys, decoding accuracy with a given number of channels was higher using spike recordings than using LFP power in either the $1-4$ or $100-170 \mathrm{~Hz}$ bands (Fig. $7 B$ ). In monkey $\mathrm{Y}$ but not monkey $\mathrm{X}$, decoding accuracies also were higher using spike recordings than using LFP amplitude in the time domain (Fig. 7A).

\section{Spatial variation in decoding accuracy}

In addition to variation dependent on movement type, each neurophysiological signal type also showed variation from electrode to electrode, as illustrated in Figures 3, 4, and 6. We therefore examined the possibility that such variations were not random but rather depended on the spatial location of recordings within
$16-24 \mathrm{~Hz}$

Coaxial cylinder

the M1 upper extremity representation. Given that for each neurophysiological signal we obtained decoding accuracies substantially greater than chance using a relatively small number of channels, we used decoding accuracy in subsets of channels to investigate the extent to which movement-type information varied depending on the spatial location of recording sites. Separate analyses were performed to examine spatiotemporal distribution down the anterior bank of the central sulcus and along the central sulcus from anterolateral to posteromedial.

\section{Distribution down the anterior bank of the central sulcus}

To examine variation down the anterior bank of the central sulcus, LDA was performed using channels grouped by depth below the hemispheric surface, as determined by the length of different electrodes on each array. In monkey $\mathrm{X}$, each array had electrodes from 1.0 to $6.0 \mathrm{~mm}$ in length, but in monkey $\mathrm{Y}$, the shortest electrodes were $1.5 \mathrm{~mm}$ long and arrays $\mathrm{H}$ and J each had five electrodes $>6.0 \mathrm{~mm}$ in length. For analysis of distribution in depth, we therefore excluded data from these 10 extra-long electrodes in monkey Y. (We subsequently repeated the analysis including data from the extra-long electrodes and obtained similar results.) Recordings in each monkey then were divided into two groups at an electrode length that provided similar numbers of electrodes in the shallow and deep groups for each monkey. In monkey $\mathrm{Y}$, the shallow group of recordings were obtained from electrodes $2.0-3.5 \mathrm{~mm}$ long, whereas the deep group were obtained from electrodes 4.0-6.0 mm long. In monkey X, the shallow group were from electrodes $1.5-3.0 \mathrm{~mm}$ long, whereas the deep group were from electrodes $3.5-6.0 \mathrm{~mm}$ long.

For each neurophysiological signal in each session, we then performed LDA as a function of time separately for the shallow and deep groups in each monkey. To permit the most direct comparison, we used the lowest common number of channels across all four signal types and depth groups: 10 for monkey $\mathrm{Y}$ and 13 for monkey $\mathrm{X}$. If for any signal type, any group had a greater number of channels available, we randomly selected the lowest common number of channels from the larger set and repeated the LDA 100 times. LDA was performed repeatedly in 50 ms time steps with data aligned separately at the time of the cue (five steps), OM (five steps), and the beginning of the SH (19 steps). In the LFP frequency domain, we examined decoding accuracy using power in the $1-4 \mathrm{~Hz}$ power and $100-170 \mathrm{~Hz}$ bands separately. Because LFP power in the frequency domain was evaluated in $250 \mathrm{~ms}$ windows, for all signal types, LDA was performed using data averaged in $250 \mathrm{~ms}$ windows centered at each $50 \mathrm{~ms}$ time step. Consequently, these analyses incorporate data up to $125 \mathrm{~ms}$ before and $125 \mathrm{~ms}$ after the nominal time point.

Figure 8 shows the time course of decoding accuracy for each neurophysiological signal (columns) in each session (rows). Sep- 
arate curves are shown for the shallow group (blue traces), the deep group (red traces), as well as for LDA performed using all available LFP channels or spike recordings (black lines, total LFP channel and spike recording counts as given in Table 1). In general, decoding accuracy rose promptly after the cue, was higher by the $\mathrm{OM}$, and achieved maximal values around the beginning of the SH. Exceptions included 1-4 Hz LFP power in monkey Y and $100-170 \mathrm{~Hz}$ power in monkey $\mathrm{X}$, in which decoding accuracy achieved its highest levels around the OM and tended to decline before the SH. Decoding accuracy generally declined after the beginning of the $\mathrm{SH}$ for all types of signal. The decline occurred earliest with 1-4 Hz LFP power, later with LFP amplitude, and tended to be only very gradual with $100-$ $170 \mathrm{~Hz}$ LFP power or spikes. Indeed in monkey $\mathrm{Y}$, decoding accuracy obtained with $100-170 \mathrm{~Hz}$ LFP power or with spikes remained at steady high levels throughout the final hold period. Although information about movement type thus appeared rapidly after the cue in all signal types, it persisted longer after the beginning of the $\mathrm{SH}$ in spikes and 100$170 \mathrm{~Hz}$ power than in $1-4 \mathrm{~Hz}$ power or LFP amplitude.

In addition to this temporal difference, movement-type information in the various neurophysiological signals also differed in spatial distribution down the anterior bank of the central sulcus. For LFP amplitude (Fig. 8A) and $1-4 \mathrm{~Hz}$ power (Fig. $8 B$ ), decoding accuracy curves for the shallow and deep groups rose and fell quite close together, although short epochs of separation were observed in some instances. Moreover, decoding accuracies obtained using either the shallow or the deep group attained values almost as high as those obtained using all available recordings. Movement-type information contained in LFP amplitude and in 1-4 $\mathrm{Hz}$ power thus was distributed quite similarly in both shallow and deep locations in the anterior bank of the central sulcus.

In contrast, decoding accuracies obtained with either $100-$ $170 \mathrm{~Hz}$ LFP power (Fig. 8C) or spike recordings (Fig. 8D) rapidly became higher for the shallow than the deep groups and remained higher throughout the movement period. In monkey X, however, decoding accuracies obtained with the shallow group of spike recordings fell below accuracies obtained with deep recordings after the beginning of the SH. In both sessions from both monkeys, using 100-170 Hz LFP power, the shallow group provided decoding accuracies comparable with those obtained using all electrodes, whereas the deep group provided substantially lower accuracies. These observations indicate that, for both 100 $170 \mathrm{~Hz}$ LFP power and spike recordings, more discriminable movement-type information was available close to the hemispheric surface than deep in the anterior bank of the central sulcus.
B C
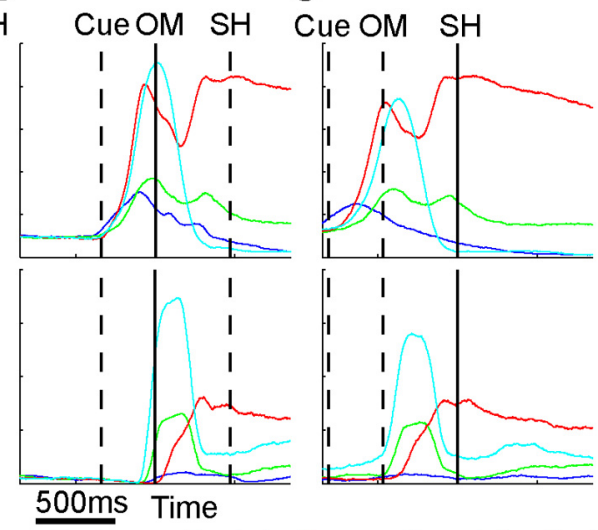

Push button

Figure 6. Variation in spike firing rates. Firing rate histograms of two SUs isolated from two different arrays (top vs bottom) in monkey $\mathrm{Y}$ are shown for each of the four movements. Data from multiple successful trials of each movement were averaged separately aligned (solid vertical line at center) on the cue $(\boldsymbol{A}), \mathrm{OM}(\boldsymbol{B})$, or SH (C). The average time of the other behavioral events is indicated by dashed vertical lines in each frame. Movement-dependent variations were prominent for each unit but also differed

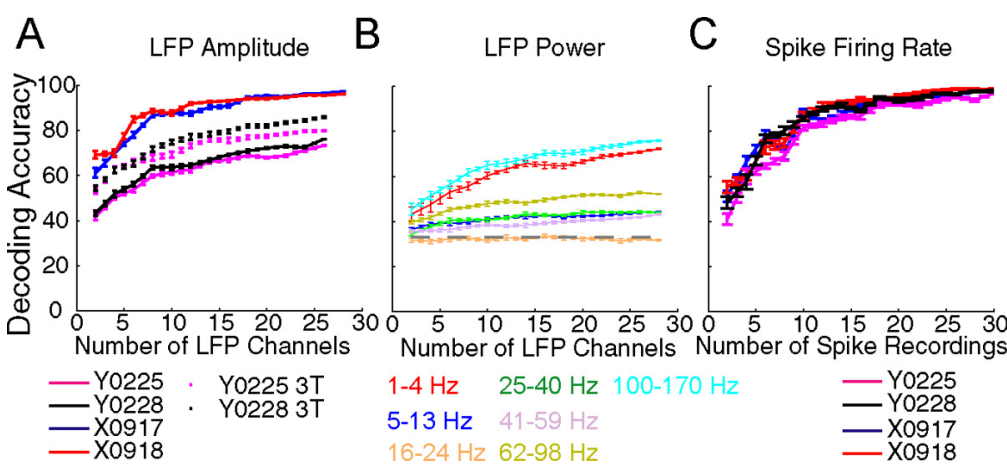

Figure 7. Decoding accuracy as a function of the number of input channels. A, LFP amplitude. In each recording session (solid accuracies were higher in monkey $X$ than monkey $Y$, even after correcting for the different number of movement types being whereas the $16-24 \mathrm{~Hz}$ band contained relatively little movement-specific information (chance level of $33 \%$ ). C Spike firing rate. accuracies were similar across all recording sessions and achieved levels similar to those obtained with LFP amplitude $(\boldsymbol{A})$. Each point represents the mean \pm SE of 20 LDAs performed with the indicated number of randomly selected LFP or spike recordings.

In addition to attaining different levels, in monkey $\mathrm{X}$, decoding accuracies obtained by the shallow and deep groups using $100-170 \mathrm{~Hz}$ LFP power also showed substantially different temporal evolution. In both sessions, decoding accuracies obtained with monkey X's shallow group rose rapidly after the cue, reached maximal values near the OM, fell somewhat by the time of $\mathrm{SH}$, and remained relatively flat thereafter. In contrast, decoding accuracies obtained with monkey X's deep group rose slowly after the cue, did not reach maximal values until near the time of $\mathrm{SH}$, and fell more rapidly thereafter. Although less dramatic, decoding accuracies obtained with monkey X's spike recordings also rose faster with the shallow group than with the deep group and fell faster after the beginning of the $\mathrm{SH}$ as well. Such differences in the temporal evolution of decoding accuracy were not observed in monkey Y. We speculate that these differences between the two monkeys might have been related to the tendency of monkey $\mathrm{X}$ to react and to move more quickly than monkey Y. 


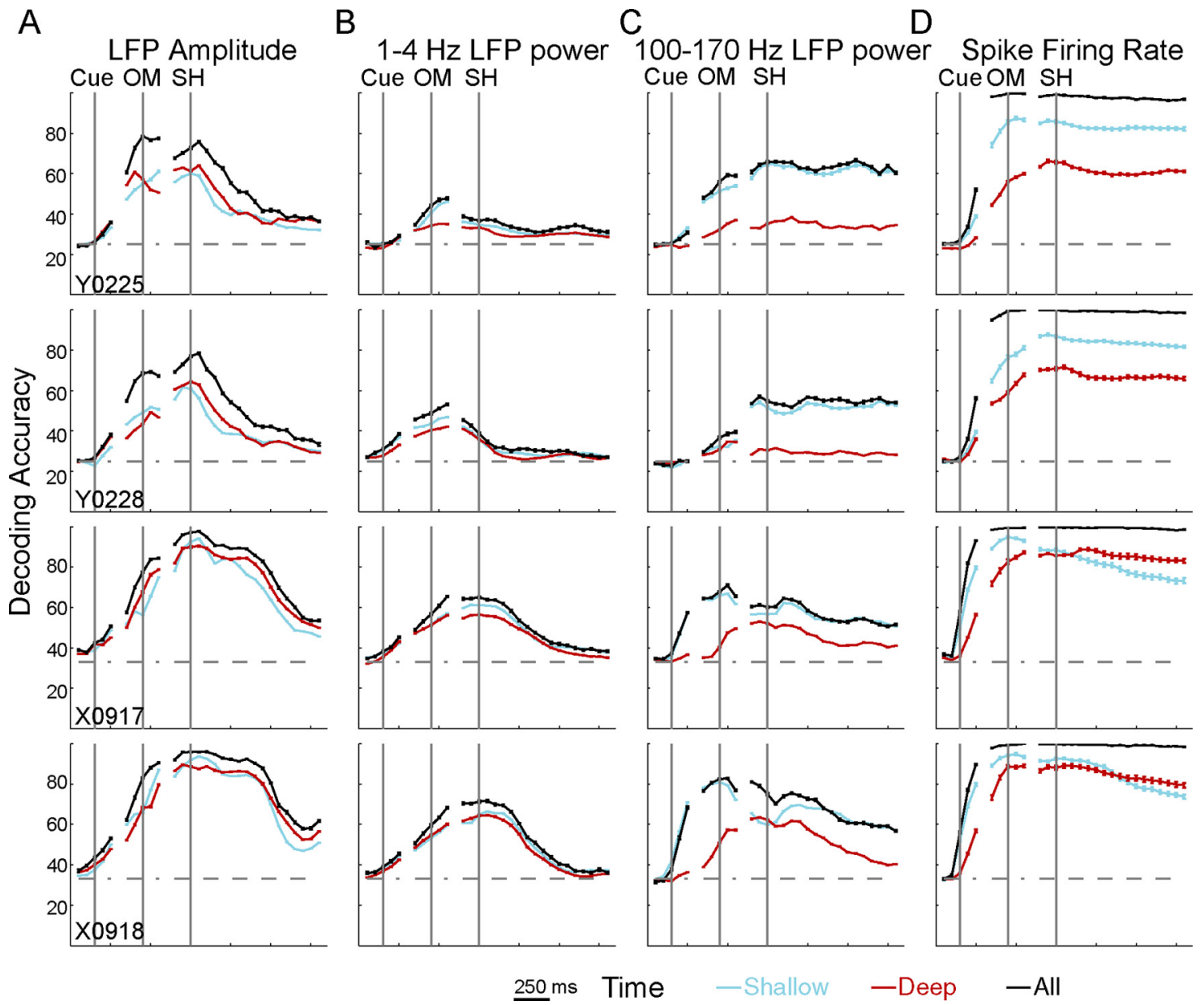

Figure 8. Spatiotemporal modulation of neural activity in depth down the anterior bank of the central sulcus. Equal numbers of channels were assigned to shallow (blue lines) and deep (red lines) groups based on the length of the recording electrodes (see Results). For each neurophysiological signal type (columns $\boldsymbol{A}-\boldsymbol{D}$ ), LDA was performed separately for the shallow and deep group and repeated in $50 \mathrm{~ms}$ steps with data from each recording session (rows) aligned separately on cue, $0 \mathrm{M}$, and SH. Black lines represent the results of similar analyses using all available channels for each signal type. Each point represents the mean \pm SE across 100 random selections of the lowest common number of recordings across groups. Movement-related information was evenly distributed down the anterior bank of the central sulcus for LFP amplitude $(\boldsymbol{A})$ and LFP power in the $1-4 \mathrm{~Hz}$ band $(\boldsymbol{B})$ but was stronger in the shallow group than in the deep group for $100-170 \mathrm{~Hz}$ power $(\boldsymbol{C})$ and spikes (D).

To further quantify the effect of depth down the anterior bank, we performed two-way ANOVA using time and depth as factors. Two-way ANOVAs were performed separately for decoding accuracies aligned at each of the three behavioral events (cue, OM, and $\mathrm{SH}$ ) for each of the four types of neurophysiological signal (LFP amplitude, 1-4 Hz LFP power, 100-170 Hz LFP power, and spikes), in each of the four sessions, totaling $48(=3 \times 4 \times 4)$ two-way ANOVAs. The time factor had five categories (50 ms time steps) for cue-aligned ANOVAs, five categories for OM-aligned ANOVAs, and 19 for SH-aligned ANOVAs. (The last time point of the SHaligned data was nominally $800 \mathrm{~ms}$ after the beginning of the $\mathrm{SH}$ and thus incorporated data up to $925 \mathrm{~ms}$ after $\mathrm{SH}$, with the hold period lasting $1000 \mathrm{~ms}$.) The depth factor had two categories, shallow and deep, for each ANOVA. In all 48 ANOVAs, the main effect of time, the main effect of depth, and the time $X$ depth interaction all were significant $(p<0.00001$ or $p<0.0015$ after Bonferroni's correction for $48 \times 3$ tests), indicating that decoding accuracy varied with time, with depth, and that the variation with time depended on depth in all cases. Shallow versus deep differences thus were significant even when the difference was relatively small, as for 1-4 Hz LFP power aligned on the cue (Fig. $8 B$ ).

We therefore examined the size of the depth effect by calculating $\eta^{2}$ for the depth factor in each two-way ANOVA (Stark et al., 2007). Effect size, $\eta^{2}$, was calculated as the ratio of the sumof-squares variance in decoding accuracy attributable to depth to the total sum-of-squares variance and expressed as a percentage. Values of $\eta^{2}$ for depth are shown in Table 2 for data aligned at each of the three behavioral events using each neurophysiological signal type in each session. With few exceptions, the effect of depth was greatest on decoding accuracies obtained using 100$170 \mathrm{~Hz}$ power and spikes, still less with $1-4 \mathrm{~Hz}$ power, and least with LFP amplitude. Movement-type information thus was distributed relatively evenly to shallow and deep locations with LFP amplitude and 1-4 Hz power, but less information reached deep locations with $100-170 \mathrm{~Hz}$ power or spikes.

\section{Distribution along the central sulcus}

We also used decoding accuracy in subsets of channels to investigate the extent to which movement-type information varied depending on the spatial location of recording sites along the central sulcus. For this analysis, LDA was performed using channels grouped by their parent array. Because array I in monkey $\mathrm{Y}$ had only five useable LFP channels, to permit accurate comparisons only five channels from each array were used in an LDA. To evenly sample all the channels, therefore, the five channels used from the other three arrays were chosen randomly 100 times, and 
Table 2. Effect size $\left(\eta^{2}\right)$ of depth category on the decoding accuracy of different neural signals around different task events

\begin{tabular}{|c|c|c|c|c|c|c|c|c|c|c|c|c|}
\hline & \multicolumn{3}{|c|}{ LFP amplitude } & \multicolumn{3}{|c|}{ LFP power at $1-4 \mathrm{~Hz}$} & \multicolumn{3}{|c|}{ LFP power at $100-170 \mathrm{~Hz}$} & \multicolumn{3}{|c|}{ Spike firing rate } \\
\hline & Cue & OM & SH & Cue & $0 \mathrm{M}$ & SH & Cue & $0 \mathrm{M}$ & SH & Cue & $0 \mathrm{M}$ & SH \\
\hline Y0225 & 1 & 8 & 1 & 5 & 34 & 8 & 28 & 93 & 99 & 25 & 89 & 98 \\
\hline Y0228 & 11 & 1 & 5 & 8 & 60 & 3 & 34 & 24 & 98 & 3 & 66 & 95 \\
\hline X0917 & 2 & 7 & 1 & 5 & 1 & 3 & 21 & 87 & 61 & 17 & 69 & 29 \\
\hline X0918 & 1 & 7 & 1 & 7 & 2 & 1 & 31 & 87 & 42 & 16 & 47 & 1 \\
\hline Average & 4 & 6 & 2 & 6 & 24 & 4 & 29 & 73 & 75 & 15 & 68 & 56 \\
\hline
\end{tabular}

A

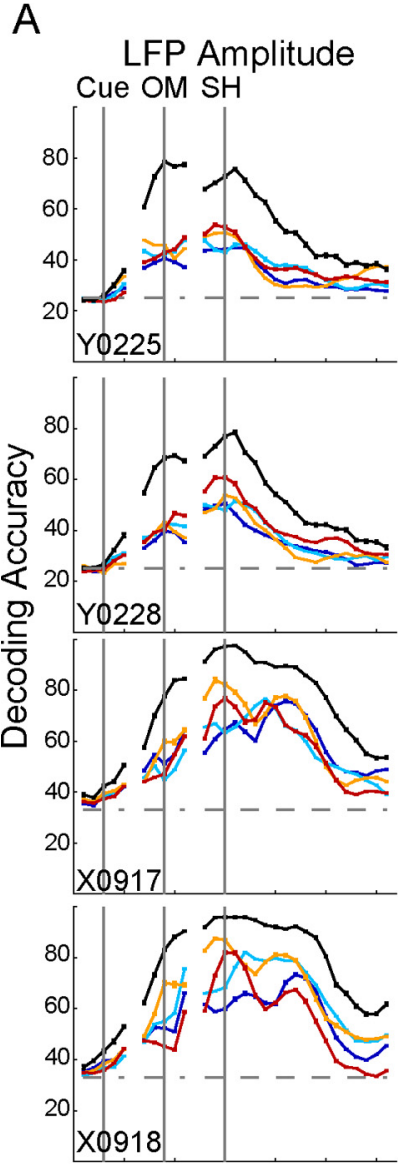

B

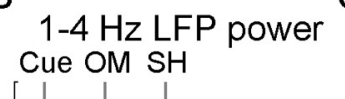

C
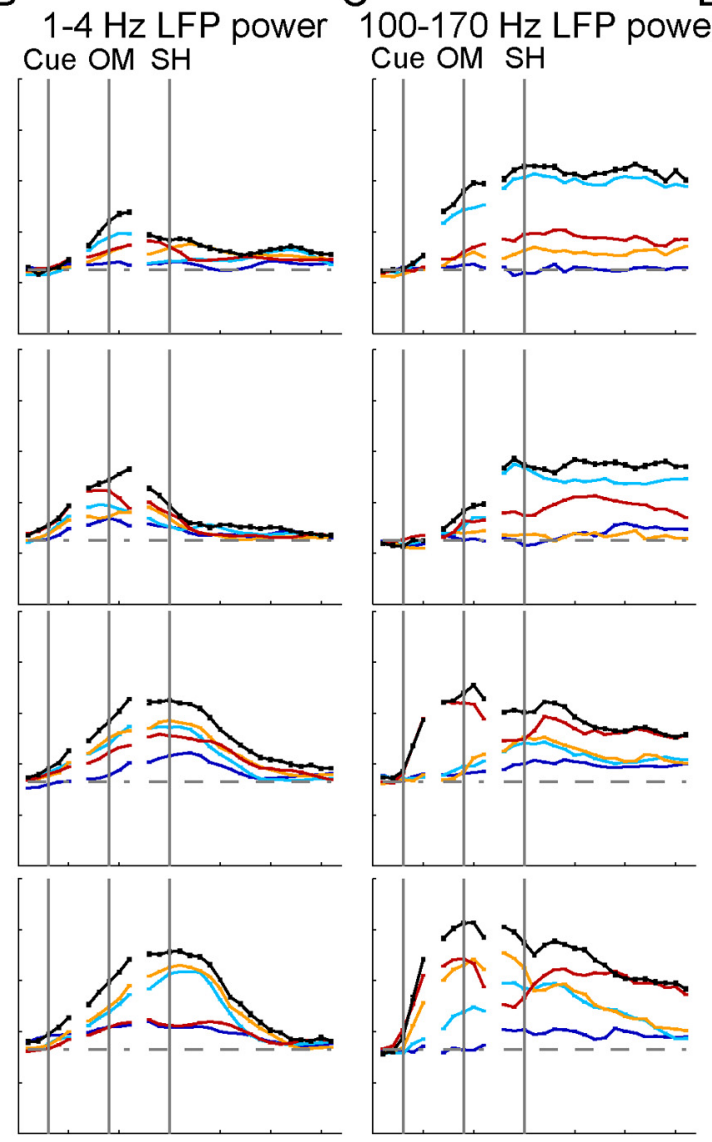

D

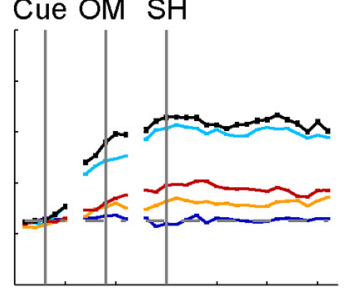

Spike Firing Rate Cue OM SH
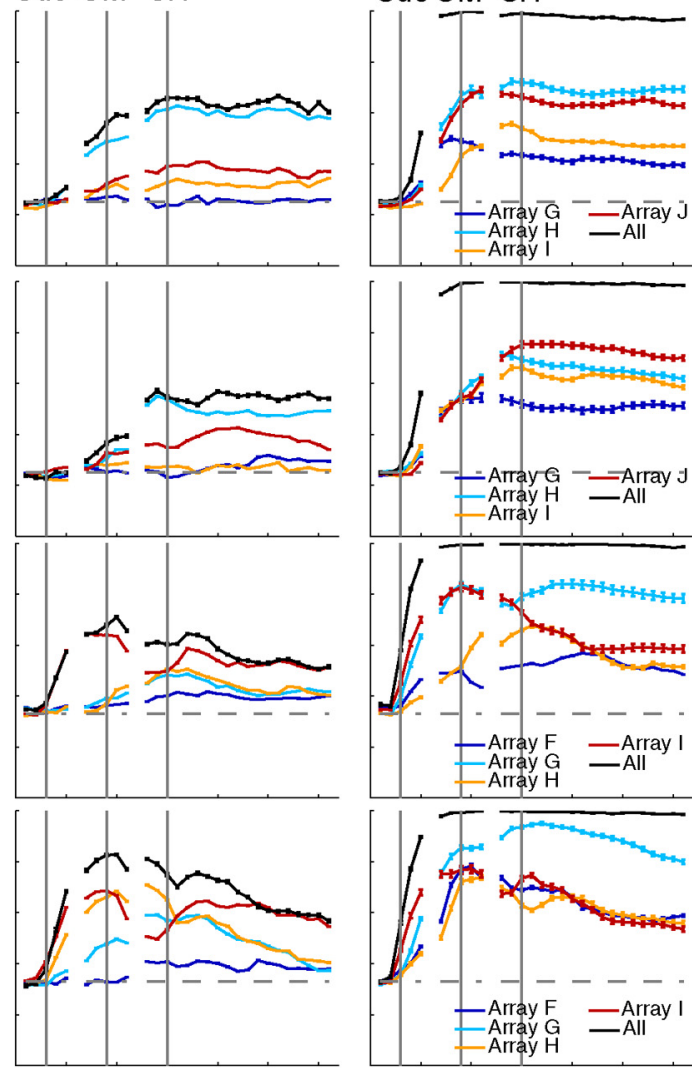

$\underline{250} \mathrm{~ms}$ Time

Figure 9. Spatiotemporal modulation of movement-related information along the central sulcus. Equal numbers of channels were grouped based on the parent array. For each neurophysiological signal type (columns $\boldsymbol{A}-\boldsymbol{D}$ ), LDA then was performed separately for each group (array) and repeated in $50 \mathrm{~ms}$ steps with data from each recording session (rows) aligned separately on cue, OM, and SH. Solid lines (identical to those in Fig. 8) represent the results of similar analyses using all available channels for each signal type. Each point represents the mean \pm SE across 100 random selections of the lowest common number of recordings across groups. Along the central sulcus, movement-related information was more evenly distributed for LFP amplitude ( $\boldsymbol{A})$ and LFP power in the $1-4 \mathrm{~Hz}$ band $(\boldsymbol{B})$ than for $100-170 \mathrm{~Hz}$ LFP power ( $\boldsymbol{C}$ and spikes (D).

the LDA was repeated for each randomly chosen set. Likewise, because array G in monkey X had only six useable LFP channels, LDA was performed for each array in monkey $\mathrm{X}$ using six channels. To evaluate temporal variation, LDA again was performed repeatedly in $50 \mathrm{~ms}$ steps with data aligned at the time of the cue (five steps), OM (five steps), and SH (19 steps).

Figure 9 shows the time course of decoding accuracy for each neurophysiological signal type in each session. Separate curves are shown for each array, as well as for LDA performed using all available LFP channels or spike recordings (Table 1). With LFP amplitude (Fig. 9A), the decoding accuracy curves for the four arrays rose and fell relatively close together, often crossing one another and showing little systematic separation of different curves across time. Movement-type information contained in
LFP amplitude thus was distributed relatively evenly along the central sulcus.

In contrast, decoding accuracy curves obtained with spike recordings (Fig. 9D) tended to separate and remain separated for the four arrays, particularly in monkey $\mathrm{Y}$, indicating that different levels of movement-type information were present at different locations along the central sulcus (Fig. 9D). In monkey $\mathrm{Y}$, arrays $\mathrm{H}$ (cyan) and $\mathrm{J}$ (red) tended to have the highest decoding accuracy, followed by array I (orange), with array G (blue) having the lowest. In monkey X, array I (red) showed a time-dependent variation, having decoding accuracy as high as that of array $\mathrm{G}$ (cyan) before the OM but falling close to the level of array $\mathrm{H}$ (orange) after the beginning of the SH. In monkey $\mathrm{X}$, spike firing rates at different locations along the 
Table 3. Effect size $\left(\eta^{2}\right)$ of array on the decoding accuracy of different neural signals around different task events

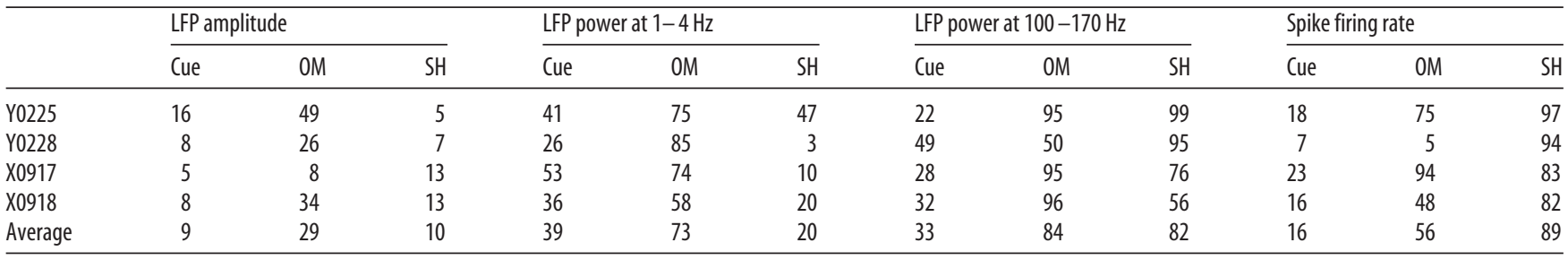

central sulcus thus provided different levels of movementtype information at different times.

In the LFP frequency domain, array-dependent variation in decoding accuracy using $1-4 \mathrm{~Hz}$ power was intermediate between that seen with LFP amplitude and that seen with spike recordings (Fig. 9B), whereas array-dependent variation in decoding accuracy using $100-170 \mathrm{~Hz}$ power was similar to that seen with spike recordings (Fig. 9C). With $1-4 \mathrm{~Hz}$ power, the decoding accuracy curves tended to separate somewhat before the OM and maintain their relative order through the $\mathrm{SH}$. The most lateral array in each monkey (array G in monkey $\mathrm{Y}$, array $\mathrm{F}$ in monkey X) provided the lowest decoding accuracy. With 100$170 \mathrm{~Hz}$ power, the curves for different arrays separated before the $\mathrm{OM}$ and maintained their separation through the SH. Overall, movement-type information transmitted by LFP power thus was more dependent on array location in the $100-170 \mathrm{~Hz}$ band than in the $1-4 \mathrm{~Hz}$ band.

To better quantify these observations, we performed two-way ANOVA using time and array as factors. These analyses were similar to the time $\times$ depth ANOVAs performed above, but here the array factor had four categories for each ANOVA. In all 48 ANOVAs, each main effect and the time $X$ array interaction all were significant $(p<0.00001$ or $p<0.0015$ after Bonferroni's correction for $48 \times 3$ tests), confirming that decoding accuracy varied with time, with array, and that the variation with time depended on the array in all cases.

We then calculated $\eta^{2}$ for the array factor to quantify the percentage of the variation in decoding accuracy attributable to array location for data aligned at each of the three behavioral events using each neurophysiological signal type in each session. Values of $\eta^{2}$ shown in Table 3 confirm that the effect of array location along the central sulcus was generally greatest for spike recordings and 100-170 Hz LFP power, less for 1-4 Hz power, and least for LFP amplitude. In all sessions except X0917, spike recordings showed the greatest effect of array location around the SH. LFP power in the $100-170 \mathrm{~Hz}$ band showed comparable array location effects around the $\mathrm{OM}$ and $\mathrm{SH}$, whereas the other two signal types tended to show their greatest array effect around the OM. Although discriminable information on movement type thus was distributed relatively evenly by LFP amplitude, with $100-170 \mathrm{~Hz}$ power or spikes different locations along the central sulcus showed different levels of movement-type information.

\section{Discussion}

Our observations are consistent with previous studies that have reported relationships between reach direction and M1 LFP activity. Time domain mEPs and low-frequency $(1-4 \mathrm{~Hz})$ and high-frequency $(>60 \mathrm{~Hz})$ power all show directional tuning during reaching movements (Rickert et al., 2005; Heldman et al., 2006), with the low-frequency burst beginning time-locked to the visual instructional cue (cf. Fig. 3A), producing an instruction evoked potential (O'Leary and Hatsopoulos, 2006). LFP power in mid-frequency bands $(15-50 \mathrm{~Hz})$ typically drops before the OM and recovers during SH. During stable holds, 15-30 Hz LFP power shows selectivity for grasp (Spinks et al., 2008). Our observations suggest in addition that the $1-4$ and $100-170 \mathrm{~Hz}$ bands show movement-type selectivity during reaction and movement times, as well as during the final, stable hold.

Furthermore, because we implanted arrays over a comparatively large M1 territory, we were able to examine the spatiotemporal distribution of neural activity during reach-to-grasp. Although early studies using optical imaging of M1 in the more lissencephalic Cebus monkey have suggested some degree of modular regionalization during reaching movements (Reinert and Strick, 2010), given that, during the present movements, the entire upper extremity was in motion simultaneously (Jeannerod, 1984; Paulignan et al., 1990; Mason et al., 2001, 2004), we expected to find a relatively uniform distribution of discriminable movementtype information. Such was not the case, however, either in depth down the anterior bank or along the central sulcus.

\section{Distribution in depth down the anterior bank of the central sulcus}

Dividing the recordings from each monkey into shallow and deep groups at $3.0-3.5 \mathrm{~mm}$ from the hemispheric surface revealed that discriminable information was distributed evenly in depth for LFP amplitude and for 1-4 Hz power but differentially for $100-$ $170 \mathrm{~Hz}$ LFP power and for spike recordings. For LFP amplitude and 1-4 Hz power, similar levels of decoding accuracy were obtained using the shallow or deep group of recordings, and those levels were comparable with the decoding accuracy obtained using all available features for those two signal types, indicating that the two groups contained redundant movement-type information. For $100-170 \mathrm{~Hz}$ LFP power and spike recordings, however, decoding accuracy was higher for the shallow group of recordings than for the deep, indicating that the deep group had less information on movement type.

Two factors may have contributed to this difference in discriminable movement-type information between shallow and deep recordings. First, the transition between Brodmann's area 4 and area 3 a occurs in the depth of the central sulcus. Although SUs related to individuated finger movements typically are recorded at depths up to $6 \mathrm{~mm}$ down the anterior bank (Schieber and Hibbard, 1993; Poliakov and Schieber, 1999; Schieber and Rivlis, 2005), the transition from area 4 to area 3a may occur at depths as shallow as $4 \mathrm{~mm}$ (Park et al., 2001; Rathelot and Strick, 2006). Our deep group thus might have included a substantial fraction of recordings from area $3 \mathrm{a}$, which despite the strong proprioceptive input to area $3 \mathrm{a}$, might not be as informative about movement type as $100-170 \mathrm{~Hz}$ power or spike recordings from area 4 . That the shallow and deep groups provided comparable decoding accuracies using either LFP amplitude or $1-4 \mathrm{~Hz}$ power then would suggest that these signals extended across the boundary between area 4 and area 3 a.

A second factor contributing to the effect of depth may be rostrocaudal regionalization within area 4 . Squirrel monkeys 
have spatially separate rostral and caudal M1 zones; within each zone, ICMS evokes digit movements more caudally and wrist movements more rostrally (Strick and Preston, 1982a). Under anesthesia, the rostral zone receives somatosensory input primarily from deep muscle and joint receptors, whereas in the caudal zone, somatosensory input is primarily cutaneous (Strick and Preston, 1982b). In macaque monkeys, rostral, intermediate, and caudal divisions can be distinguished anatomically within area 4 (Preuss et al., 1997). Although segregation of somatosensory inputs may not follow these regional boundaries in awake macaques (Wong et al., 1978; Lemon, 1981), the caudal portion of M1 in the anterior bank of the central sulcus contains the corticomotoneuronal (CM) cells that make monosynaptic connections to spinal $\alpha$-motoneurons, whereas the rostral portion on the crown of the precentral gyrus contains few CM cells (Rathelot and Strick, 2009). Whereas rostral M1 neurons show strong relationships to movement kinematics (Moran and Schwartz, 1999a,b; Schwartz and Moran, 1999), caudal M1 neurons show strong relationships to forces and movement dynamics (Kalaska et al., 1989; Sergio et al., 2005). In humans, the posterior region of area 4 shows more functional activation during motor imagery and attention to action than does the anterior region (Binkofski et al., 2002; Sharma et al., 2008). These regional differences may reflect underlying functional differences between rostral and caudal regions within $\mathrm{M} 1$.

Our deep group of recordings was likely to have sampled entirely from the caudal region of $\mathrm{M} 1$, whereas our shallow recordings included sites on the crown of the precentral gyrus extending anteriorly up to $\sim 2.5 \mathrm{~mm}$ away from the sulcus per se and thus sampled substantially from the rostral region of M1 as well. Our finding that $100-170 \mathrm{~Hz}$ LFP power and spike recordings in the shallow group contained more information on movement type than the deep group therefore suggests that the rostral region of M1 may play a larger role in control of reach-to-grasp movements than the caudal region. Recent work showing that neurons related to grasp shape are found in the more rostral region of M1 (Hendrix et al., 2009) and that complete reach-to-grasp movements can be decoded from recordings limited to $2 \mathrm{~mm}$ in depth from the hemispheric surface (Vargas-Irwin et al., 2010) would be consistent with this notion.

\section{Distribution along the central sulcus}

Dividing the recordings from each monkey into groups by array allowed us to examine the spatial distribution of discriminable movement-type information along the central sulcus. As with depth, consistent variation of decoding accuracy related to array location along the central sulcus was least evident with LFP amplitude, somewhat more apparent with 1-4 Hz power, and greatest with 100-170 $\mathrm{Hz}$ power or spike recordings. This array-dependent variation could have resulted from chance differences in the placement of arrays. We note, however, that in both monkeys the highest decoding accuracies using spike recordings generally were obtained using the arrays in the ICMS-defined medial shoulder region (Fig. 9D, red curves) and the more lateral digit core (Fig. 9D, cyan curves). The variation we observed along the central sulcus thus might have resulted from differential involvement of the shoulder, elbow, wrist, and digits in the present task: the shoulder movements used to reach to different locations and the digit movements used to grasp different objects varying more with movement type than the elbow movements used to flex and extend the forearm or the wrist movements used to orient the hand.

In summary, we found regional variation in the spatial distribution of decodable movement-type information within M1, both in depth down the anterior bank of the central sulcus and along the sulcus. Our findings do not enable us, however, to reliably attribute different functions to different locations within M1. The present task did not distinguish whether the modulation of neurophysiological signals decoded here might have resulted from sensory inputs, such as different proprioceptive inputs from the various grasp postures and/or different tactile inputs from contact of different parts of the palm and fingers with the various objects, nor did the present task dissociate the reach location produced by proximal musculature from the grasp shape produced by distal musculature (Asher et al., 2007; Stark et al., 2007). Understanding the extent to which the regional variation in movement-type information described here reflects regional differences in sensory input to and motor output from M1 will require additional studies.

\section{Movement representation in low-frequency versus high-frequency LFP power}

We found that LFP amplitude or 1-4 Hz power spread discriminable movement-type information more evenly through the M1 upper extremity representation than $100-170 \mathrm{~Hz}$ LFP power or spikes. The similarity between LFP amplitude and 1-4 Hz power likely reflects the predominant contribution of these low frequencies to mEPs. The other two signal types studied here-100$170 \mathrm{~Hz}$ power and spike firing rates- have been observed to show parallel modulation in both the secondary somatosensory area (Ray et al., 2008) and the middle temporal area (Liu and Newsome, 2006). Together, these observations suggest that two distinct mechanisms distribute movement-type information in M1 during reach-to-grasp.

These mechanisms may not be entirely independent, however. Like the present findings, human electrocorticography has shown a progressively more focal distribution of oscillatory activity modulation proceeding from lower $(8-13 \mathrm{~Hz} \alpha$ and $15-25$ $\mathrm{Hz} \beta$ ) to higher $(35-50 \mathrm{~Hz}$ low gamma and $75-100 \mathrm{~Hz}$ high gamma) frequencies (Crone et al., 1998a,b; Miller et al., 2007), and studies in macaque auditory cortex have suggested that the amplitude of higher-frequency oscillations may be modulated by the phase of lower-frequency oscillations (Lakatos et al., 2005). Widespread distribution of low-frequency signals thus might be sculpted progressively to more focally modulated high-frequency signals.

\section{Movement decoding for brain-machine interfaces}

Our findings have a number of implications for movement decoding in brain-machine interface applications. The more widespread distribution of LFP amplitude and 1-4 Hz power suggest that these signals may be the most useful if a limited area within the M1 upper extremity representation is used for decoding reach-to-grasp movements (Bansal et al., 2011). Although good decoding accuracy can be achieved during reaction and movement periods using these two signal types, decoding accuracy declines during stable holds. Decoding accuracy using either $100-170 \mathrm{~Hz}$ LFP power or spike firing rates is better maintained during stable holds but may require more widely distributed sampling in the more rostral region of M1. Optimal decoding of reach-to-grasp therefore might be obtained by combining multiple neurophysiological signals.

\section{References}

Asher I, Stark E, Abeles M, Prut Y (2007) Comparison of direction and object selectivity of local field potentials and single units in macaque 
posterior parietal cortex during prehension. J Neurophysiol 97:36843695.

Bansal AK, Vargas-Irwin CE, Truccolo W, Donoghue JP (2011) Relationships among low-frequency local field potentials, spiking activity, and three-dimensional reach and grasp kinematics in primary motor and ventral premotor cortices. J Neurophysiol 105:1603-1619.

Binkofski F, Fink GR, Geyer S, Buccino G, Gruber O, Shah NJ, Taylor JG, Seitz RJ, Zilles K, Freund HJ (2002) Neural activity in human primary motor cortex areas $4 \mathrm{a}$ and $4 \mathrm{p}$ is modulated differentially by attention to action. J Neurophysiol 88:514-519.

Chestek CA, Cunningham JP, Gilja V, Nuyujukian P, Ryu SI, Shenoy KV (2009) Neural prosthetic systems: current problems and future directions. Conf Proc IEEE Eng Med Biol Soc 2009:3369-3375.

Crone NE, Miglioretti DL, Gordon B, Sieracki JM, Wilson MT, Uematsu S, Lesser RP (1998a) Functional mapping of human sensorimotor cortex with electrocorticographic spectral analysis. I. Alpha and beta eventrelated desynchronization. Brain 121:2271-2299.

Crone NE, Miglioretti DL, Gordon B, Lesser RP (1998b) Functional mapping of human sensorimotor cortex with electrocorticographic spectral analysis. II. Event-related synchronization in the gamma band. Brain 121:2301-2315.

Graziano MS, Taylor CS, Moore T (2002) Complex movements evoked by microstimulation of precentral cortex. Neuron 34:841-851.

Heldman DA, Wang W, Chan SS, Moran DW (2006) Local field potential spectral tuning in motor cortex during reaching. IEEE Trans Neural Syst Rehabil Eng 14:180-183.

Hendrix CM, Mason CR, Ebner TJ (2009) Signaling of grasp dimension and grasp force in dorsal premotor cortex and primary motor cortex neurons during reach to grasp in the monkey. J Neurophysiol 102:132-145.

Jeannerod M (1984) The timing of natural prehension movements. J Mot Behav 16:235-254.

Kalaska JF, Cohen DA, Hyde ML, Prud'homme M (1989) A comparison of movement direction-related versus load direction-related activity in primate motor cortex, using a two-dimensional reaching task. J Neurosci 9:2080-2102.

Kwan HC, MacKay WA, Murphy JT, Wong YC (1978) Spatial organization of precentral cortex in awake primates. II. Motor outputs. J Neurophysiol 41:1120-1131.

Lakatos P, Shah AS, Knuth KH, Ulbert I, Karmos G, Schroeder CE (2005) An oscillatory hierarchy controlling neuronal excitability and stimulus processing in the auditory cortex. J Neurophysiol 94:1904-1911.

Lemon RN (1981) Functional properties of monkey motor cortex neurones receiving afferent input from the hand and fingers. J Physiol 311:497-519.

Liu J, Newsome WT (2006) Local field potential in cortical area MT: stimulus tuning and behavioral correlations. J Neurosci 26:7779-7790.

Mallat S, Zhang Z (1993) Matching pursuit with time-frequency dictionaries. IEEE Trans Signal Proc 41:3397-3415.

Mason CR, Gomez JE, Ebner TJ (2001) Hand synergies during reach-tograsp. J Neurophysiol 86:2896-2910.

Mason CR, Theverapperuma LS, Hendrix CM, Ebner TJ (2004) Monkey hand postural synergies during reach-to-grasp in the absence of vision of the hand and object. J Neurophysiol 91:2826-2837.

Miller KJ, Leuthardt EC, Schalk G, Rao RP, Anderson NR, Moran DW, Miller JW, Ojemann JG (2007) Spectral changes in cortical surface potentials during motor movement. J Neurosci 27:2424-2432.

Moran DW, Schwartz AB (1999a) Motor cortical representation of speed and direction during reaching. J Neurophysiol 82:2676-2692.

Moran DW, Schwartz AB (1999b) Motor cortical activity during drawing movements: population representation during spiral tracing. J Neurophysiol 82:2693-2704.

Murthy VN, Fetz EE (1996) Synchronization of neurons during local field potential oscillations in sensorimotor cortex of awake monkeys. J Neurophysiol 76:3968-3982.

Musallam S, Bak MJ, Troyk PR, Andersen RA (2007) A floating metal microelectrode array for chronic implantation. J Neurosci Methods 160:122-127.

O’Leary JG, Hatsopoulos NG (2006) Early visuomotor representations revealed from evoked local field potentials in motor and premotor cortical areas. J Neurophysiol 96:1492-1506.
Park MC, Belhaj-Saif A, Gordon M, Cheney PD (2001) Consistent features in the forelimb representation of primary motor cortex in rhesus macaques. J Neurosci 21:2784-2792.

Paulignan Y, MacKenzie C, Marteniuk R, Jeannerod M (1990) The coupling of arm and finger movements during prehension. Exp Brain Res 79:431-435.

Penfield W, Boldrey E (1937) Somatic motor and sensory representation in the cerebral cortex of man as studied by electrical stimulation. Brain 37:389-443.

Poliakov AV, Schieber MH (1999) Limited functional grouping of neurons in the motor cortex hand area during individuated finger movements: a cluster analysis. J Neurophysiol 82:3488-3505.

Preuss TM, Stepniewska I, Jain N, Kaas JH (1997) Multiple divisions of macaque precentral motor cortex identified with neurofilament antibody SMI-32. Brain Res 767:148-153.

Rathelot JA, Strick PL (2006) Muscle representation in the macaque motor cortex: an anatomical perspective. Proc Natl Acad Sci U S A 103:82578262.

Rathelot JA, Strick PL (2009) Subdivisions of primary motor cortex based on cortico-motoneuronal cells. Proc Natl Acad Sci U S A 106:918-923.

Ray S, Crone NE, Niebur E, Franaszczuk PJ, Hsiao SS (2008) Neural correlates of high-gamma oscillations $(60-200 \mathrm{~Hz})$ in macaque local field potentials and their potential implications in electrocorticography. J Neurosci 28:11526-11536.

Reinert KC, Strick PL (2010) Evidence for a modular organization in M1 and PMd based on patches of activation during reaching movements. Soc Neurosci Abstr 36:83.5.

Rickert J, Oliveira SC, Vaadia E, Aertsen A, Rotter S, Mehring C (2005) Encoding of movement direction in different frequency ranges of motor cortical local field potentials. J Neurosci 25:8815-8824.

Schieber MH, Hibbard LS (1993) How somatotopic is the motor cortex hand area? Science 261:489-492.

Schieber MH, Rivlis G (2005) A spectrum from pure post-spike effects to synchrony effects in spike-triggered averages of electromyographic activity during skilled finger movements. J Neurophysiol 94:3325-3341.

Schwartz AB, Moran DW (1999) Motor cortical activity during drawing movements: population representation during lemniscate tracing. J Neurophysiol 82:2705-2718.

Sergio LE, Hamel-Pâquet C, Kalaska JF (2005) Motor cortex neural correlates of output kinematics and kinetics during isometric-force and armreaching tasks. J Neurophysiol 94:2353-2378.

Sharma N, Jones PS, Carpenter TA, Baron JC (2008) Mapping the involvement of BA 4a and 4p during motor imagery. Neuroimage 41:92-99.

Spinks RL, Kraskov A, Brochier T, Umilta MA, Lemon RN (2008) Selectivity for grasp in local field potential and single neuron activity recorded simultaneously from M1 and F5 in the awake macaque monkey. J Neurosci 28:10961-10971.

Stark E, Asher I, Abeles M (2007) Encoding of reach and grasp by single neurons in premotor cortex is independent of recording site. J Neurophysiol 97:3351-3364.

Strick PL, Preston JB (1982a) Two representations of the hand in area 4 of a primate. I. Motor output organization. J Neurophysiol 48:139-149.

Strick PL, Preston JB (1982b) Two representations of the hand in area 4 of a primate. II. Somatosensory input organization. J Neurophysiol $48: 150-159$.

Theverapperuma LS, Hendrix CM, Mason CR, Ebner TJ (2006) Finger movements during reach-to-grasp in the monkey: amplitude scaling of a temporal synergy. Exp Brain Res 169:433-448.

Vargas-Irwin CE, Shakhnarovich G, Yadollahpour P, Mislow JM, Black MJ, Donoghue JP (2010) Decoding complete reach and grasp actions from local primary motor cortex populations. J Neurosci 30:9659-9669.

Wong YC, Kwan HC, MacKay WA, Murphy JT (1978) Spatial organization of precentral cortex in awake primates. I. Somatosensory inputs. J Neurophysiol 41:1107-1119.

Woolsey CN, Settlage PH, Meyer DR, Sencer W, Hamuy TP, Travis AM (1952) Patterns of localization in precentral and "supplementary" motor areas and their relation to the concept of a premotor area. Res Publ Assoc Res Nerv Ment Dis 30:238-264. 